\title{
Distale Bizepssehnen- und Trizepssehnenrupturen
}

Florian Freislederer, Donato Papillo, Michael Glanzmann, Markus Scheibel

\section{Rupturen der distalen Bizeps- und Trizepssehne sind selten, treten jedoch gehäuft bei männlichen, sportlichen und körperlich schwer arbeitenden Patienten auf. Dieser Übersichtsartikel beschreibt Ätiologie und Pathogenese sowie Diagnostik, Behand- lungsmöglichkeiten, das zu erwartende Outcome dieser Verletzungen und gibt Ein- blicke in das eigene Vorgehen.}

$\begin{array}{ll}\text { ABKÜRZUNGEN } \\ \text { ASES } & \begin{array}{l}\text { American Shoulder and Elbow Sur- } \\ \text { geons }\end{array} \\ \text { CRPS } & \begin{array}{l}\text { Complex regional Pain Syndrome } \\ \text { DASH Score }\end{array} \\ \text { Disability of Arm, Shoulder and Hand } \\ \text { NSAID } & \text { Nonsteroidal anti-inflammatory Drug } \\ & \text { (nichtsteroidales Antiphlogistikum) } \\ \text { PIN } & \text { Nervus interosseus posterior } \\ \text { PRP } & \text { Platelet Rich Plasma } \\ \text { ROM } & \text { Range of Motion }\end{array}$

\section{Distale Bizepssehnenruptur}

\section{Ätiologie}

Mit einer Inzidenz von 12-2,5 pro 100000 ist die distale Bizepssehnenruptur eine seltene Verletzung [1,2]. Sie macht lediglich 3-12\% aller Bizepssehnenrupturen aus und tritt gehäuft bei Männern mittleren Alters (30-50 Jahre) überwiegend an der dominanten Seite auf [1,35].

\section{Risikofaktoren}

Besondere Risikofaktoren der distalen Bizepssehnenruptur $[1,6,7]$ sind

- Kraftsport,

- anabole Steroide und

- Rauchen.

\section{Pathogenese}

Hinsichtlich der Pathogenese werden verschiedene Ursachen diskutiert. Die vaskuläre Anatomie der distalen Bizepssehne wurde untersucht und 3 verschiedene Zonen beschrieben. Der proximale, als Zone 1 bezeichnete Teil erhält eine reichliche Blutversorgung aus der $A$. brachialis. Der distalste, als Zone 3 beschriebene Teil wird durch Äste der A. interossea posterior versorgt. Die hypovasku- läre Zone 2 liegt zwischen diesen beiden Versorgungsgebieten. Mikroskopisch konnten hier degenerative Veränderungen festgestellt werden.

Einen möglichen extrinsischen Faktor stellt der mechanische Konflikt bei Umwendbewegungen dar. In vollständiger Pronation halbiert sich die Distanz zwischen Ulna und Tuberositas radii. Außerdem wird bei pronierter Stellung dieser Raum zum großen Teil von der Bizepssehne ausgefüllt $[5,8,9]$.

Eames et al. beschreiben in einer Kadaverstudie einen möglichen Zusammenhang zwischen Unterarmflexion und distaler Bizepsschädigung. Bei Kontraktur der Unterarmflexoren vergrößert sich ihre Querschnittsfläche. Dies spannt die Aponeurose (Lacertus fibrosus) und übt Zug nach medial auf die Bizepssehne aus [10].

\section{Anatomie und Biomechanik}

Im Gegensatz zur Schulter ist die Integrität und Funktion des M. biceps brachii am Ellenbogen als wichtigster Supinator und, neben dem M. brachialis, wichtiger Beuger von großer Bedeutung.

Analog zur Trennung proximal besteht auch die distale Bizepssehne aus einem kurzen und einem langen Anteil. Aus einer koronaren Ausrichtung dreht die Sehne nach außen in eine sagittale Ebene am Ansatz. Am muskulotendinösen Übergang ist das Caput brevis medial. Im Verlauf dreht es sich $90^{\circ}$ nach außen und inseriert an der Tuberositas radii distal des Caput longum, entfernt von der Rotationsachse des Ellenbogens $[5,11]$ ( $\bullet$ Abb.1a, Abb. 1 b). Somit wird postuliert, dass die kurze Bizepssehne eher für die Flexion verantwortlich ist. Die lange Bizepssehne setzt proximaler und posterior, weiter entfernt von der Rotationsachse des Radius, an und wird daher eher als Supinator gesehen [12]. 

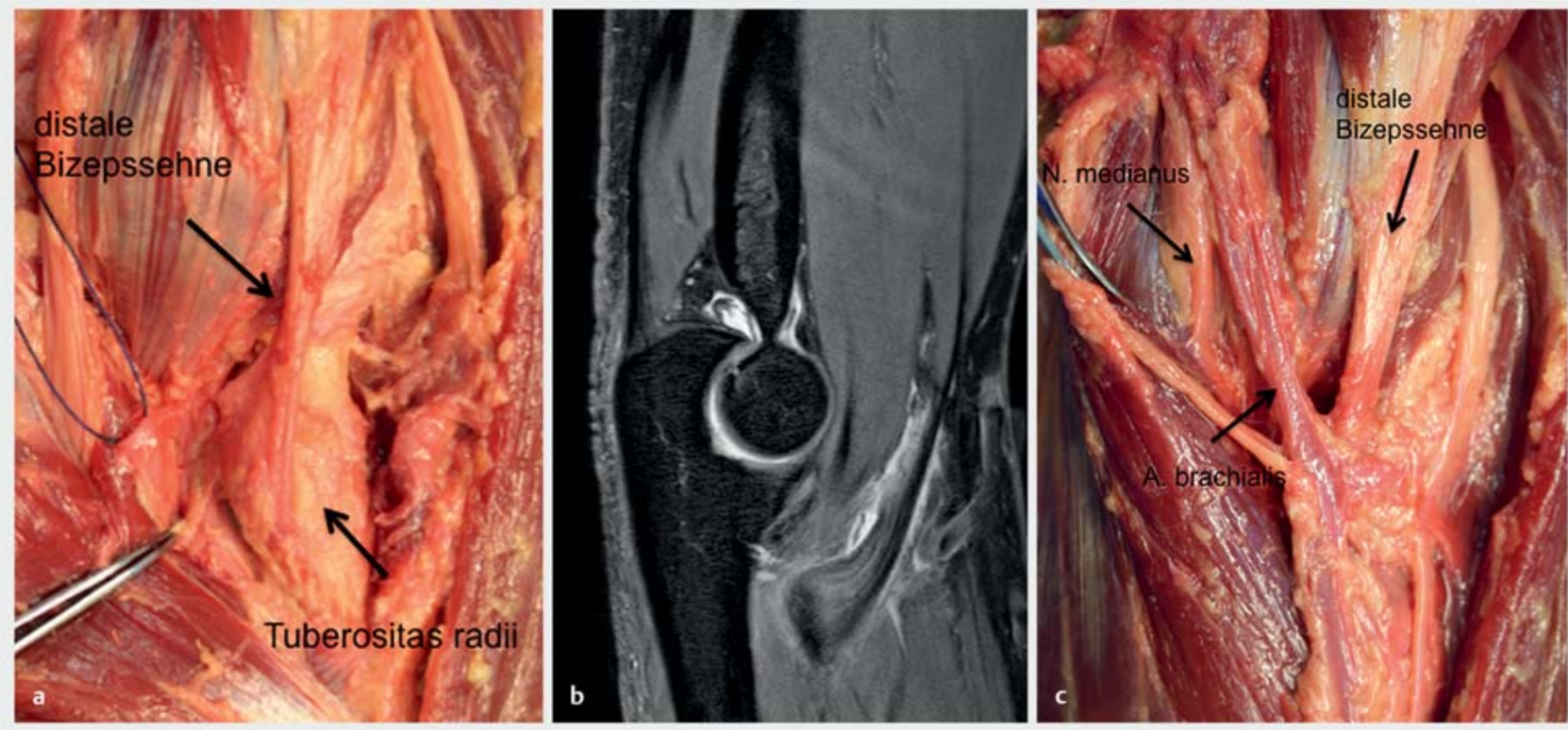

- Abb. 1 a Rotation der distalen Bizepssehne mit Insertion der medialen Anteile distal und der lateralen proximal und posterior. b Sagittale MRTSchicht eines rechten Ellbogens: Partialläsion mit erhaltenem medialem Footprint. Sichtbare Rotation der medialen Sehnenanteile mit fächerförmigem Ansatz. c Anatomie der Antekubitalregion. (Quelle a u. c: Gerhardt C, Scheibel M. Chirurgische und funktionelle Anatomie. In: Müller L, Hollinger B, Burkhart K, Hrsg. Expertise Ellenbogen. 1. Auflage. Stuttgart: Thieme; 2016. doi:10.1055/b-003-128229).

Die Aponeurosis bicipitalis, besser bekannt als Lacertus fibrosus, entspringt im Bereich der kurzen Bizepssehnenanteile, strahlt medial in die Unterarmfaszie ein und inseriert an der Ulna. Der Lacertus fibrosus schützt die neurovaskulären Strukturen und lenkt Flexionskräfte ulnarseitig. Der Aufbau des Lacertus ist dreischichtig. Diese Schichten umschlingen Pulley-ähnlich die beiden Anteile der distalen Bizepssehne und sind mit ihnen verbunden. Somit wurde eine mögliche Funktion als Stabilisator der distalen Bizepssehne beschrieben [10].

Die Integrität dieser Struktur ist ein wichtiges Hindernis für eine proximale Migration des Muskelbauches bei chronischen Rupturen [3,10].

Neurovaskuläre Strukturen befinden sich im Zugangsgebiet und in unmittelbarer Nähe zur Sehne. Oberflächlich zwischen M. biceps brachii und M. brachialis ist der N. cutaneus antebrachii lateralis zu beachten. Der N. interosseus posterior (tiefer Ast des N. radialis, engl. PIN) taucht auf Höhe des Radiushalses im Bereich der FrohseArkade durch den M. supinator in das dorsale Vorderarmkompartiment. Die A. und V. brachialis sowie der N. medianus befinden sich medialseitig der Bizepssehne ( Abb. 1c).

\section{Klinik}

Anamnese

Traumatische Rupturen sind meist durch eine plötzliche exzentrische Belastung bedingt. Ein Sturz auf den gestreckten Arm, Ellenbogenextension beim Kraftsport oder abrupte Extension des in Supination gehaltenen Vorderarms sind typische Mechanismen.

Oft wird von einer reißenden, schmerzhaften Sensation in der Ellenbeuge und einem hörbaren Schnappen berichtet. Die Bildung eines Hämatoms in der Antekubitalregion und die genaue Schmerzlokalisation sind variabel. Komplette Rupturen mit Verletzung des Lacertus fibrosus sind durch die Retraktion des Muskelbauches mit dem sog. Reverse-Popeye-Zeichen bei Ellenbogenflexion gut zu erkennen ( $\triangleright$ Abb. $\mathbf{2 a}$ a). Bei Partialrupturen, aber auch bei Totalrupturen mit intaktem Lacertus fibrosus, ist oft keine Retraktion erkennbar.

\footnotetext{
Merke

Im Vordergrund steht die schmerzhafte, teils auch schmerzlose Krafteinschränkung in Supination und Flexion.
}

\section{Klinische Untersuchung}

Zur weiterführenden Befunderhebung sind diverse klinische Tests entwickelt worden. 
Hook-Test: Der Hook-Test gilt als pathognomonisch für eine Ruptur [13]. Ist die distale Bizepssehne intakt und wird der Patient gebeten, bei $90^{\circ}$ flektiertem Ellenbogen eine Supination aktiv durchzuführen, kann der Untersucher seinen Zeigefinger unter der Sehne von der lateralen Seite aus einhaken ( $\triangleright$ Abb. $\mathbf{2}$ b).

\section{Merke}

Das digitale Testen von lateral ist wichtig, da medial ein intakter Lacertus fibrosus das Ergebnis verfälschen kann.

Die Sensitivität und Spezifität dieses Tests wurde von der erstbeschreibenden Gruppe um O’Driscoll mit 100\% angegeben.

Bizeps-Squeeze-Test: Ein weiterer diagnostischer Test, ähnlich dem Thompson-Test zur Diagnose von Rupturen der Achillessehne, ist der Bizeps-Squeeze-Test. Hierbei hält der sitzende Patient seinen Arm entspannt bei ca. $60^{\circ}$ Flexion und Pronation im Schoß, und der Untersucher komprimiert den Oberarm. Hierdurch sollte bei intakter distaler Bizepssehne eine Supination des Unterarms hervorgerufen werden. Dieser Test ist zu 96\% sensitiv, bei jedoch weitestgehend unbekannter Spezifität [14].

Biceps-Crease-Test: Der Biceps-Crease-Test nach ElMaraghy et al. misst das Intervall zwischen Ellenbogenfalte und distalem Ende des Bizepsmuskelbauches in cm und stellt es mit der gesunden Gegenseite ins Verhältnis. Mit einem Intervall $>6 \mathrm{~cm}$ oder einem Verhältnis zur Gegenseite $\geq 1,2$ geben die Autoren eine Sensitivität von $96 \%$ und Spezifität von 93\% für die Diagnose einer distalen Bizepssehnenruptur an [15].

Supination-Pronation-Test: Metzman beschreibt, vor allem zur schnellen Diagnose von Totalrupturen im Notfallsetting, den Supination-Pronation-Test. Hierbei wird lediglich die distale Kontur des Bizepsbauches bei aktiven Pro-/Supinationsbewegungen betrachtet. Bei intakter Sehne proximalisiert sich der Muskelbauch bei Supination und distalisiert sich bei Pronation. Bei fehlender Bewegung ist der Test als positiv zu werten [16].

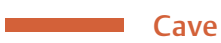

Bei Partialrupturen können die zuvor erwähnten klinischen Manöver unauffällig bleiben.

\section{TIPP}

Aus eigener Erfahrung empfiehlt es sich bei Verdacht auf eine Partialläsion der distalen Bizepssehne, die resistive Supinationsprüfung nicht nur in $90^{\circ}$ Ellenbogenflexion, sondern auch in stärker gebeugtem Gelenk, ca. bei $120^{\circ}$ durchzuführen. Oft äußert dann

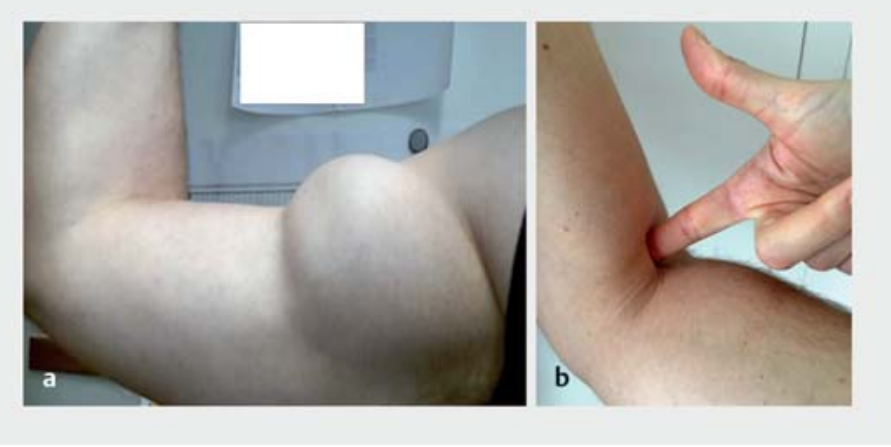

- Abb. 2 Klinik bei distaler Bizepssehnenruptur. a Reverse Popeye Sign mit deutlicher Retraktion des Muskels in Flexion als Zeichen einer distalen Bizepssehnenruptur. b Hook-Test nach O'Driscoll. In der Abbildung ( $\mathrm{OA}=$ Oberarm, UA = Unterarm) zeigt sich ein nicht pathologischer Befund; die Sehne kann von lateral her mit dem Untersucherfinger unterfahren werden.

der Patient die Wahrnehmung des für ihn typischen Schmerzes mit oder ohne Kraftverlust.

\section{Bildgebung}

Als Standardbildgebung wird ein konventionelles Röntgenbild des Ellbogens in 2 Ebenen durchgeführt, um ossäre Läsionen, knöcherne Ausrisse und Veränderungen im Bereich der Tuberositas radii zu detektieren.

Die Ultraschalldiagnostik kann die Sehne bis zur Insertion auch dynamisch darstellen, ist unmittelbar verfügbar und kostengünstig. Nachteilig ist die Untersucherabhängigkeit. Der Verlauf lässt sich gut von ventral in der Ellenbeuge darstellen. Distal kann das Insertionsgebiet am besten in voller Pronation von posteroradial sowohl im Längsschnitt als auch im Horizontalschnitt gesehen werden [17] ( $\triangleright$ Abb. 3 a und b).

Merke

Goldstandard ist die MRT-Diagnostik.

Die Rupturart, Retraktion und Muskeltrophik können mit der MRT sehr gut beurteilt werden ( $\bullet$ Abb. 3c) [18]. Die ideale Position des Patientenarms zur Beurteilung des distalen Sehnenverlaufes ist in Flexion, Abduktion und Supination (FABS-Position), da hierbei in einer Schnittebene der gesamte Verlauf der Sehne vom muskulotendinösen Übergang bis zur Insertion dargestellt werden kann [19]. Auch postoperativ kann mit dieser Sequenz ein „Gapping“ bei Versagen der Rekonstruktion gut evaluiert werden.

Im Falle von Partialläsionen kann die Integrität der distalen Sehneninsertion MR-tomografisch weitgehend intakt erscheinen. Ein peritendinöses Serom ist in solchen 

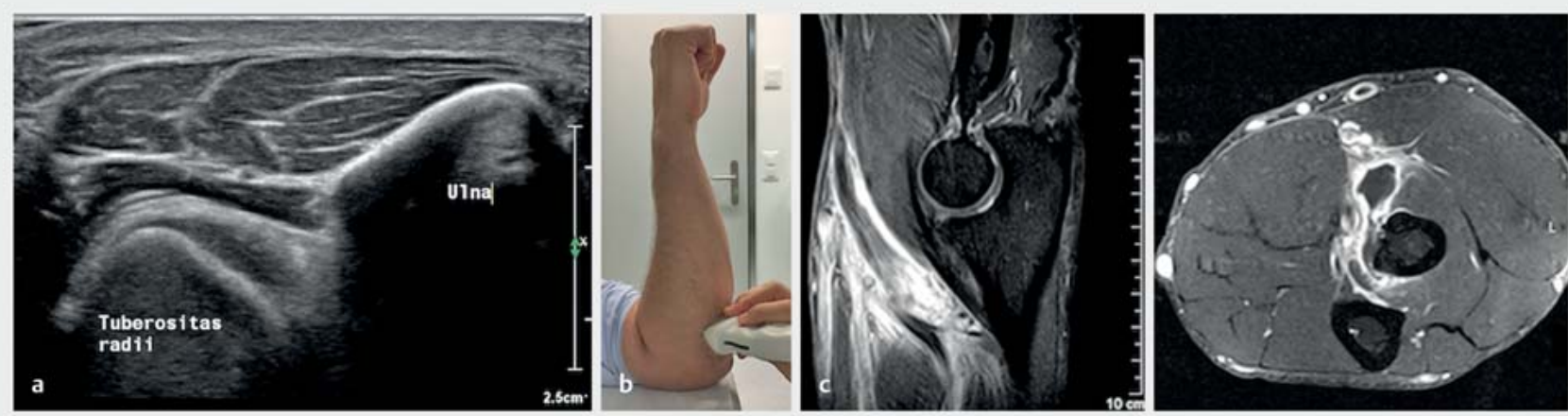

- Abb. 3 a Der Bizepssehnenansatz in Pronation mittels Ultraschallbildgebung von dorsal in horizontaler Schnittebene. b Position des Schallkopfs und Armhaltung. c Sagittale und axiale Schicht eines MRT mit Totalruptur ohne Retraktion.

Fällen ein hartes Indiz für die korrekte, klinisch gestellte Verdachtsdiagnose.

\section{Konservative Therapie}

Partialrupturen, die weniger als $50 \%$ der Sehne ausmachen, werden zunächst konservativ behandelt (s.a. unten). Ein chirurgisches Débridement oder eine Rekonstruktion sind eine Option, wenn die konservative Therapie versagt. Es besteht jedoch kein Konsens darüber, welche Behandlungsmodalität angewandt oder wie lange eine konservative Behandlung versucht werden sollte, bevor eine Operation indiziert ist.

Wenn der Teilriss mehr als 50\% der Sehne beträgt, wird eher eine chirurgische Rekonstruktion empfohlen [20].

Besteht eine Totalruptur, ist die Operationsindikation gegeben, da ansonsten mit einer Kraftreduktion der Supination von bis zu $50 \%$ und der Flexion von bis zu $30 \%$ zu rechnen ist. Bei älteren Patienten mit geringem funktionellem und kosmetischem Anspruch, polymorbiden oder medizinischen Hochrisikopatienten wird im eigenen Vorgehen die konservative Therapie bevorzugt eingesetzt. Überdies muss der Patient bei seiner Entscheidungsfindung in Hinsicht auf eine mögliche Ablehnung chirurgischer Maßnahmen aufgeklärt werden.

\section{Ergebnisse}

Bei repetitiven Bewegungen wurden Abnahmen der Supinationsausdauer beschrieben [21].

Zu den Ergebnissen der konservativen Behandlung distaler Bizepssehnenrupturen liegen nur wenige Daten vor. Nesterenko et al. konnten in ihrem Patientenklientel, verglichen mit der gesunden Gegenseite, eine deutliche Reduktion der Kraft, jedoch keine Abnahme der Ausdauer feststellen [22].

Freeman et al. untersuchten 20 konservativ therapierte distale Bizepssehnenrupturen nach 38 Monaten und ver- glichen sie mit einer historischen, operativ versorgten Kontrollgruppe. Die nicht operierten Patienten erreichten gute Ergebnisse im Mayo Elbow Score (95 Punkte von 100 möglichen Punkten) und DASH-Fragebogen (9 Punkte), jedoch bestand ein signifikanter Unterschied der Supinationskraft im Vergleich zur Gegenseite und Kontrollgruppe [21].

\section{Operative Therapie}

Prinzipiell können die Operationstechniken in anatomische oder extraanatomische Rekonstruktionen eingeteilt werden. Die anatomischen Verfahren können erneut in die direkte Rekonstruktion mittels Refixation der Sehne (hauptsächlich in akuten Fällen) und die Rekonstruktion mittels Allograft (hauptsächlich in chronischen Fällen) unterteilt werden. Des Weiteren muss grundsätzlich zwischen anatomischem Vorgehen in Single- oder DoubleInzisions-Technik unterschieden werden.

Die extraanatomische Rekonstruktion beinhaltet die Naht an die Sehne des M. brachialis, um die Flexionsstärke in chronischen Fällen teilweise wiederherzustellen. Eine Wiederherstellung der Supinationskraft ist aufgrund des fehlenden Ansatzes am Hebelarm des proximalen Radius nicht möglich.

\section{Merke}

Extraanatomische Rekonstruktionen werden selten eingesetzt; eine anatomische Rekonstruktion ist aufgrund der besseren klinischen Ergebnisse anzustreben [23].

\section{Fixationstechniken}

Verschiedene Fixierungsmethoden stehen zur Verfügung. Traditionell wurden transossäre Nähte verwendet. Zu den neueren Methoden gehören

- Fadenanker,

- Endobutton und

- Interferenzschrauben. 
In zahlreichen biomechanischen Studien wurde die Reißfestigkeit dieser Techniken sowohl unter Einzellast als auch zyklischen Tests verglichen [24-31]. Die höchste maximale Versagenslast, sowohl nach zyklischer als auch nach einmaliger Belastung, hat der Endobutton (440 N), gefolgt von Fadenankern $(381 \mathrm{~N})$ und transossären Nähten $(310 \mathrm{~N})$. Interferenzschrauben stellten sich als schwächstes Konstrukt heraus (232 N) [26]. Das Versagen stellte sich in Form von Nahtrissen, Anker- oder Schrauben-Pull-out, Sehnenrupturen oder -ausrissen und Radiusfrakturen im Bereich der Tuberositas radii dar $[25,26]$.

\section{Operationstechnik: Single-Inzision}

Bei dieser Technik erfolgt der Zugang rein ventral im Sinne eines modifizierten Zugangs nach Henry. Aufgrund minimalinvasiver Methoden und der Weiterentwicklung der zur Verfügung stehenden Implantate konnte das Risiko neurovaskulärer Verletzungen gesenkt werden [34].

Über einen S-förmigen Hautschnitt von ca. $5 \mathrm{~cm}$ Länge wird unter Aufsuchen und Schützen des N. cutaneus antebrachii lateralis in die Tiefe entlang der Bursa bicipitoradialis auf die Tuberositas radii präpariert. Die Sehne lässt sich im akuten Fall hier meist gut ertasten, mobilisieren und reponieren ( $\bullet \mathbf{A b b} \mathbf{4} \mathbf{a}$ ). In chronischen Fällen ist die Anatomie oft durch Narbengewebe verändert, und auch das Aufsuchen der retrahierten Sehne bedarf einer Erweiterung des Zugangs nach proximal. Die Querinzision sollte exakt in der Ellenbeuge gesetzt werden.

\section{THERAPIE}

\section{OP-Schritte und Tricks}

Bei bikortikalem Vorgehen ist aufgrund der Nähe des R. profundus des N. radialis auf eine Bohrung, welche nicht nach distal und leicht ulnarwärts zielt bei endgradig supiniertem Arm zu achten.

Aufgrund des Durchmessers der Bohrung ist ferner eine mittige Ausrichtung im Bereich der Tuberositas radii wichtig, um iatrogene Frakturen zu vermeiden. Beim Benützen von monokortikalen Systemen ohne das Einziehen der Sehne in den Bohrkanal (TensionSlide) wird der Footprint an der Tuberositas vorher leicht angefrischt, um die epiossäre Anheilung zu gewährleisten. Schmidt et al. empfehlen jedoch, basierend auf Ergebnissen aus Kraftprüfungen in Kadaverstudien, die für die Supination wichtige Geometrie der Tuberositas radii nicht zu verändern, da vor allem die vor dem Ansatz der Bizepssehne bestehende Protuberanz der Tuberositas radii wie ein Hypomochlion für die distale Bizepssehne bei Supinationsbewegungen fungiert [37].
Im eigenen Vorgehen wird die Fixation mittels monokortikalem Fadenanker (All Suture) oder Endobuttonkonstrukt durchgeführt.

Der Button wird ebenfalls monokortikal ( $\bullet$ Abb. 4 ) oder an der Gegenkortikalis eingebracht $[35,36]$.

McKee et al. berichten mit der Nutzung von 2 Fadenankern nach einem Follow-up von 2,5 Jahren bei $53 \mathrm{~Pa}$ tienten über keine relevante Bewegungseinschränkung und einen DASH-Score von 8,2 Punkten, ohne signifikanten Unterschied zur Kontrollgruppe (6,2 Punkte) [34].

John et al. geben für eine Gruppe von 53 Patienten nach Fadenankerrefixation und einem Follow-up von 38 Monaten einen Andrews-Carson Elbow Score von 194,3 an. Hier bestanden bei 2 Patienten Defizite in der Umwendbewegung [38].

Greenberg et al. untersuchten 14 ihrer Patienten mit bikortikalem Endobutton nach einem Zeitraum von 20 Monaten und stellten $97 \%$ der ursprünglichen Flexions- und $82 \%$ der Supinationskraft fest. Der Bewegungsumfang war bei allen Patienten uneingeschränkt [36].

Heinzelmann et al. beschreiben bei 31 Patienten, die mittels Tension-Slide-Endobuttonfixation und Interferenzschraube versorgt wurden, nach 2 Jahren einen verbesserten Andrews-Carson Elbow Score (mögliche Punktzahl: 150-200) von 196 im Vergleich zu 162 präoperativ und eine Wiederaufnahme der Berufstätigkeit nach durchschnittlich 6,5 Wochen postoperativ [39].

\section{Operationstechnik: Double-Inzision}

Aufgrund der zugangsbedingten Gefahr der iatrogenen neurovaskulären Verletzung und der ungenügenden Exposition des gesamten Sehnen-Footprints an der Tuberositas radii bei der rein ventralen Exposition beschrieb die Gruppe um Boyd und Anderson bereits Anfang der 60erJahre einen 2. Zugang [40]. Diese Double-Inzisions-Technik umfasst einen kleinen Schnitt (ca. $2 \mathrm{~cm}$ ) ventral, um die Sehne zu lokalisieren und zu armieren.

In supinierter Armhaltung wird eine Klemme auf die Tuberositas radii gesetzt und die Spitze mit Kontakt zur Tuberositas radii unter begleitender Pronationsbewegung nach posterolateral geführt. Hier wird nun eine separate Inzision zur Einsicht der Tuberositas radii und direkter transossärer Naht der Sehne durchgeführt.

Nachteilig ergab sich die erhöhte Inzidenz von heterotopen Ossifikationen und auch proximalen radioulnaren Synostosen durch die partielle ulnarseitige Ablösung der Membrana interossea am Periost. Um diese Komplikation zu vermeiden, modifizierten Failla et al. [41] die Technik und versetzten den Zugang etwas ulnaferner mit limitiertem Split der Extensoren und Erhalt des ulnaren Periosts. 

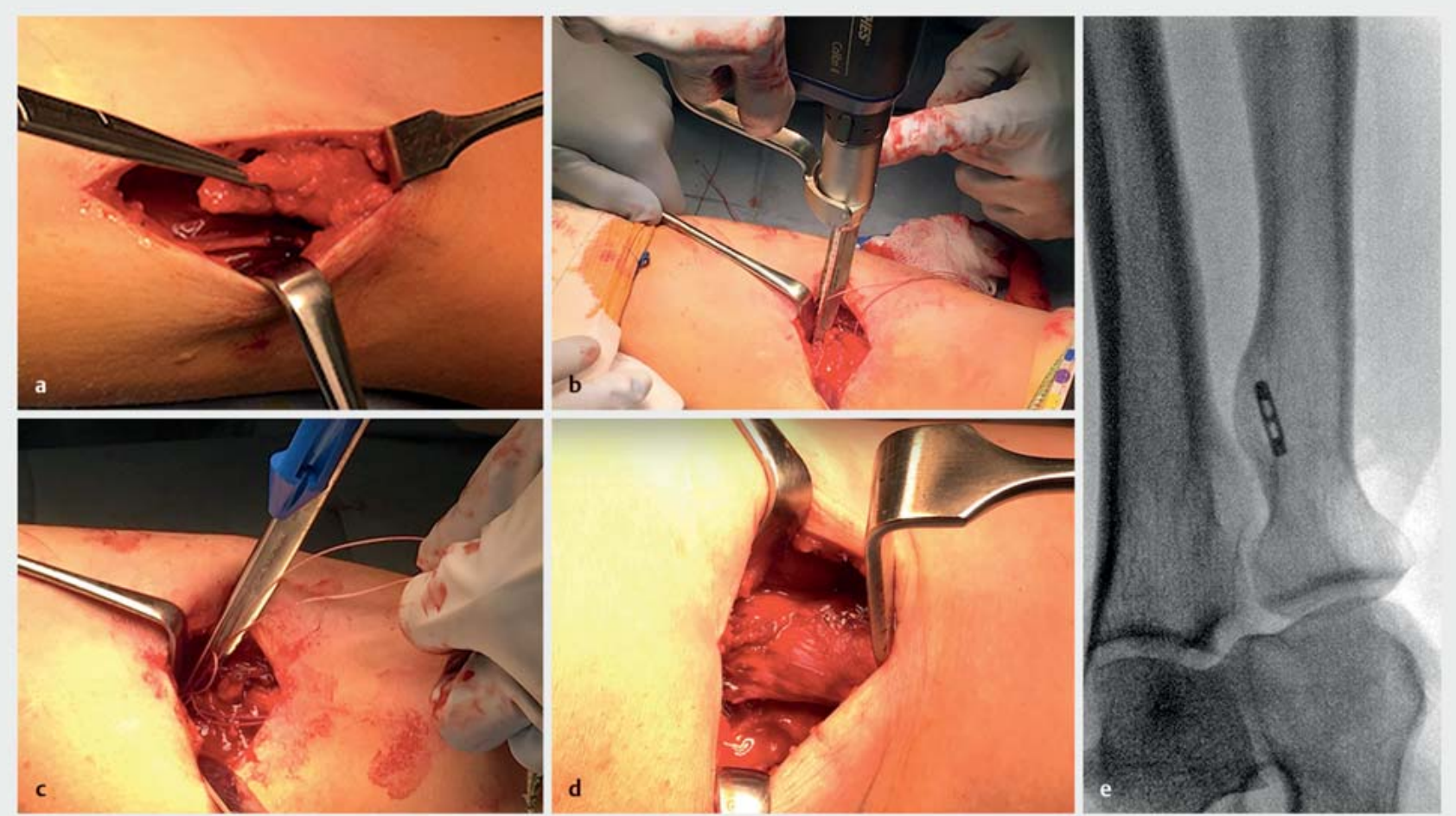

c
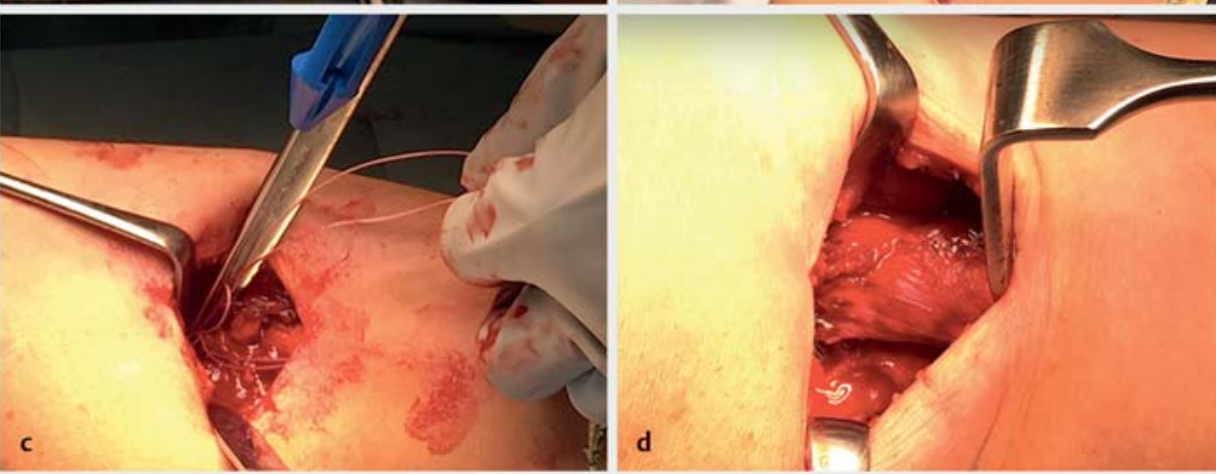

- Abb. 4 Situs einer Rekonstruktion mittels monokortikal endostal verblocktem Endobutton in Single-Inzisions-Technik über minimalinvasiven modifizierten Henry-Approach. a Darstellen der gerissenen Sehne. b Die Sehne wurde armiert, monokortikales Bohren. c Einbringen des am Faden angebrachten Endobuttons in das Bohrloch. $\mathbf{d}$ Nach Verknoten zeigt sich die rekonstruierte Sehne. e Konventionelle intraoperative Kontrolle nach Rekonstruktion über einen monokortikal endostal verblockten Endobutton.

Im Verlauf wurde jedoch auch die Bedeutung anderer Faktoren hervorgehoben, die ebenfalls mit der Entwicklung von Synostosen verbunden sein könnten, wie der Zeitpunkt des chirurgischen Repair $>2$ Wochen posttraumatisch [42].

Mehrere Studien zeigen gute Ergebnisse dieser Technik.

Nach Double-Inzision und Rekonstruktion mittels Fadenankern fanden Weinstein et al. bei 32 Patienten nach 42 Monaten keine relevante Bewegungseinschränkung und mindestens 95\% Flexions-, Supinations- und Pronationskraft verglichen mit der Gegenseite [43].

Karunkar et al. beschrieben bei ihren mit transossären Nähten versorgten 21 Patienten nach 44 Monaten bei 4 Patienten ein Defizit in der Umwendbewegung (3 in Pro- und 1 Patient in Supination), bei 1 Patient ein Extensionsdefizit (ROM 0-15-115), bei 10 Patienten eine Einschränkung der Supinationskraft, bei 3 Patienten der Flexionskraft, sowie bei 8 respektive 7 Patienten eine Verminderung der Ausdauer in Supination bzw. in Flexion. Der durchschnittliche DASH Score betrug 52,4 (Range 34-98) [44].
D’Alessandro et al. berichteten ebenfalls über transossäre Nähte bei 10 Athleten (25-49 Jahre) mit einem Follow-up von 50 Monaten [4]. Allesamt waren subjektiv zufrieden. War die nicht dominante Seite betroffen, kam es zu ca. $25 \%$ Verlust der Supinationsausdauer bei voller Flexionsund Supinationskraft und Flexionsausdauer. Die Rekonstruktion an der dominanten Extremität erbrachte volle Supinationskraft, -ausdauer und Flexionskraft, jedoch $20 \%$ Einbuße bei der Flexionsausdauer.

Single- versus Double-Inzision: anteriore versus anatomische Rekonstruktion

Die Exposition des Footprints der distalen Bizepssehne an der Tuberositas radii ist von ventral auch bei maximaler Supination aufgrund der anatomischen Verhältnisse nicht optimal, und die Tendenz, die Bohrung zu anterior durchzuführen, besteht ( $\vee$ Abb. $\mathbf{5 a}$ a). Hingegen ist die Exposition beim posterolateralen Zugang mit dem Arm in Pronation (analog zur Ultraschallschnittebene; s. - Abb. 3) gut möglich ( $\bullet$ Abb. 5 b; nach Schmidt et al. [12]).

Am Leichnam konnte gezeigt werden, dass beim ventralen Zugang nur 10\% einer 7,5-mm-Bohrung im Bereich des Footprints lagen, hingegen waren es $73 \%$ beim posterolateralen Zugang [45]. 


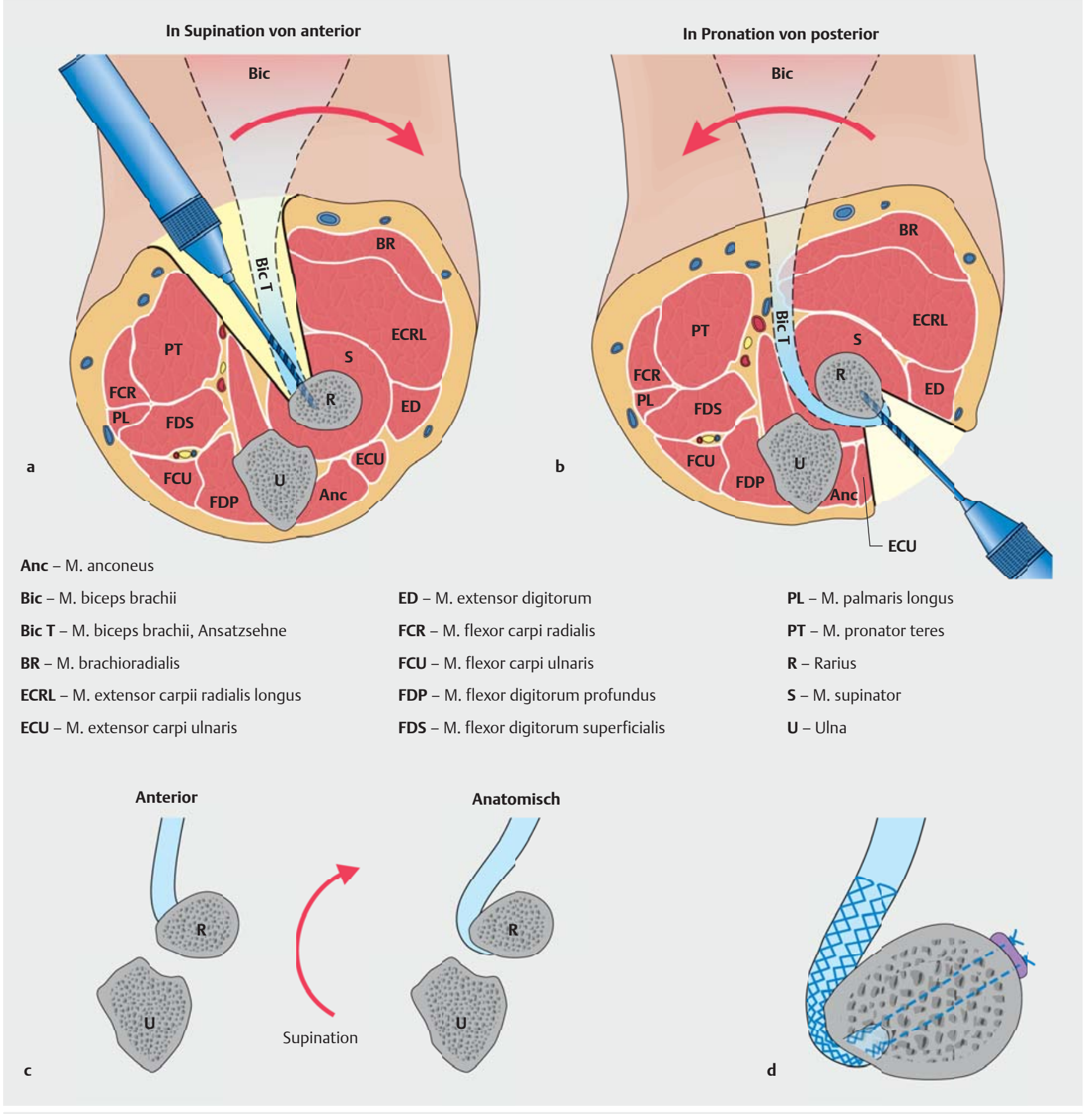

- Abb. 5 Unterschiede zwischen anteriorem und anatomischem Zugang. a Zugang in Supination von anterior. b Zugang in Pronation von posterior. Die Schwierigkeit der Bohrung im Bereich des Footprints ist beim anterioren Zugang ersichtlich. c Schwächeres Supinationsmoment der Bizepssehne (blau) bei anteriorer im Vergleich zur anatomischen Insertion. d Endoskopisch, mittels Endobutton durchgeführte Fixationstechnik.

Rekonstruktionen der Bizepssehne, die den nativen Footprint nicht angemessen wiederherstellen, können bei endgradiger Umwendbewegung keine optimale Supinationsstärke hervorbringen. Überschreitet der Unterarm die Neutralposition, verringert eine anteriore Reinsertionsplatzierung die Fähigkeit des Bizeps, ein terminales Supinationsdrehmoment herzustellen [11] ( $\mathrm{Abb} . \mathbf{5 c}$; nach [46]).
Schmidt et al. führten eine MRT-Analyse von Patienten beider Zugangsgruppen und einer Kontrollgruppe ohne Verletzung durch. Der Insertion Site Angle der Bizepssehne ISA (Winkel zwischen Spitze der Tuberositas und dem Bizepssehnenanssatzzentrum in den axialen Schichten) und der Fettgehalt des M. supinator SFC (Supinator Fat Content) wurden berechnet und verglichen. Des Weiteren wurden Kraftmessungen durchgeführt. Die Supina- 
tionskraft der posterioren Gruppe war auf der operierten Seite 19\% schwächer als auf der Gegenseite, jedoch signifikant höher als die der anterioren Gruppe. Es bestand kein signifikanter Unterschied zwischen dem ISA der posterioren und der Kontrollgruppe. Der ISA der anterioren Gruppe war jedoch signifikant höher als der ISA der Kontrollgruppe. Im Gegensatz zur anterioren Gruppe hatte die posteriore einen signifikant höheren SFC im Vergleich zur Kontrollgruppe. Erhobene funktionelle Scores (DASH) korrelierten jedoch nicht mit der Supinationskraft. Als Fazit schlussfolgerten die Autoren, dass sich zukünftige Bemühungen auf eine anatomische Insertion mit möglichst geringem Schaden des M. supinator fokussieren sollten [47].

Eine weitere Gruppe um Schmidt untersuchte die isometrische Supinationskraft in 3 verschiedenen Unterarmstellungen $\left(60^{\circ}\right.$ Supination, Neutralstellung und $60^{\circ}$ Pronation) an 23 Patienten mit kompletten Bizepssehnenrupturen und verglich diese mit der gesunden Gegenseite, um Faktoren zu entdecken, welche die Supinationskraft nach distaler Bizepssehnenruptur beeinflussen. Die verletzte Seite verlor $60 \%$ an Supinationskraft in Neutralposition. Des Weiteren war die Supinationskraft in $60^{\circ}$ Pronation stärker als in $60^{\circ}$ Supination, dies galt jedoch ebenso für die unversehrte Gegenseite. Die Autoren interpretierten die stärkere Supinationskraft aus einer pronierten Stellung bis zur Neutralen durch eine Supinationsaktivität des M. brachioradialis in dieser Bewegungsphase, ab der Neutralstellung agiert dieser als Pronator.

Cohen et al. untersuchten das funktionelle Outcome 2 Jahre nach Double- und Single-Inzisions-Rekonstruktion. 25 Patienten hatten eine Single-Inzisions- und 33 eine Double-Inzisions-Rekonstruktion mittels Endobutton erhalten. Unabhängig von der Technik waren $72 \%$ subjektiv sehr zufrieden, die verbliebenen ebenfalls zufrieden. Es fanden sich keine signifikanten Unterschiede im DASH-Score. Die Komplikationsinzidenz war vergleichbar [48].

Auch Shields et al. kamen bei 20 Single-Inzisions-Rekonstruktionen mit kortikalem Button und Interferenzschraube versus 21 Double-Inzisions-Rekonstruktionen mittels transossärem Tunnel zu ähnlichen Ergebnissen bezüglich der Patientenzufriedenheit. Auch bezüglich Bewegungsausmaß und Kraft fanden sich keine Unterschiede [49].

In einer prospektiv-randomisierten Studie von Grewal et al. wurden 47 Patienten mittels Single-Inzision und 2 Fadenankern und 44 Patienten mit Double-Inzision und transossären Nähten behandelt. Nach 2 Jahren ergaben sich keine signifikanten Unterschiede im ASES und DASH Score, sowie im Patient-Related Elbow Evaluation-Score. Auch die Kraftmessung ergab vergleichbare Ergebnisse. Patienten mit Double-Inzision profitierten verglichen mit der gesunden Seite signifikant bei der Flexion, und Patienten mit Single-Inzision erlitten signifikant häufiger temporäre Neurapraxien des N. cutaneus antebrachii lateralis. Im Fazit ihrer Arbeit konnten die Autoren diese marginalen Unterschiede jedoch vernachlässigen und schlussfolgerten, dass keine Technik der anderen bezüglich des Outcomes überlegen ist [50].

\section{Nachbehandlung}

Ein Konsens bezüglich postoperativem Bewegungslimit, Orthesen und Wiederaufnahme von Sport und körperlicher Arbeit besteht nicht.

Generell wird die Extremität direkt postoperativ bis zur Weichteilkonsolidierung in einer Oberarmschiene ruhiggestellt und dann auf eine dynamisch einstellbare Ellenbogenorthese für 6 Wochen gewechselt. Die Extension wird für 4-6 Wochen bei ca. $20-30^{\circ}$, je nach initialer Spannung der Rekonstruktion intraoperativ, limitiert.

Indomethacin wird zur Prophylaxe der Entwicklung von heterotopen Ossifikationen in einigen Ländern gegeben. In Deutschland wird das Medikament nicht mehr eingesetzt. Neueste Erkenntnisse stellen die Ossifikationsprophylaxe generell in Frage. Hudson et al. untersuchten in einer 2018 präsentierten Metaanalyse 146 Patienten, die zwischen 2008 und 2015 einen Double-Incision Repair erhielten, und kamen zum Ergebnis, dass durch Indomethacin keine Reduktion der heterotopen Ossifikationen nachzuweisen war. Es wurden sogar paradoxerweise bei den Prophylaxepatienten mehr (13,5\%) heterotope Ossifikationen gesehen als bei den Patienten ohne Prophylaxe $(5,5 \%)$ [51].

\section{Komplikationen}

Grundsätzlich haben beide Operationstechniken eigene Komplikationsmuster.

Die Gesamtkomplikationsrate nach chirurgischem Repair beträgt 15-30\% [52].

10-15\% dieser Vorkommnisse betreffen neurologische Komplikationen wie temporäre Neurapraxien des N. cutaneus antebrachii lateralis oder Parästhesien des oberflächlichen sensiblen Astes des N. radialis, vor allem beim ventralen Zugang durch Hakenzug. Grewal berichtet in seiner Studie über eine Neurapraxie des N. cutaneus antebrachii lateralis bei $44 \%$ der Patienten [50]. Besonders bei chronischen Rupturen oder Revisionen ist eine Präparation und Darstellung des Nervs zu empfehlen.

Selten kommt es zu Schäden des R. profundus des $N$. radialis. Dieser kann von posteroradial bei der DoubleInzisions-Technik verletzt werden, ist jedoch auch von ventral besonders beim Bohren, beim Einführen eines auf der posterioren Gegenkortikalis angebrachten Endo- 
buttons und beim Hakeneinsatz um den Radiushals in Gefahr.

Je nach Literatur werden in 0-50\% der Fälle heterotope Ossifikationen beschrieben.

Traditionell war die radioulnare Synostose das häufigste und am meisten beeinträchtigende ungünstige Ereignis nach der Double-Inzisions-Technik mit einer Rate von 9\%. Wie bereits erwähnt, hat die Modifikation des Zugangs unter Schonung des ulnaren Periosts diese Rate gesenkt. Das Risiko einer Reruptur beträgt 1-5\% und Bewegungseinschränkungen treten in ca. $4 \%$ der Fälle auf. Selten sind die Entwicklung eines CRPS (2\%) und Infektionen $(0-2 \%)$. Frakturen sind kaum in der Literatur beschrieben, stellen aber insbesondere bei der Verwendung einer Interferenzschraube eine potenzielle Gefahr da, die bei Eintreten schwierig zu behandeln ist.

Cain et al. untersuchten retrospektiv 198 Patienten, von denen 188 mittels Single-Inzision versorgt worden waren. Als geringwertigere Komplikationen beschreiben sie bei $26 \%$ Neurapraxien des N. cutaneus antebrachii lateralis und bei $6 \%$ des N. radialis superficialis sowie bei $2 \%$ oberflächliche Wundinfektionen. Relevante Komplikationen stellten die Schädigung des tiefen Astes des N. radialis bei $4 \%$, symptomatische heterotope Ossifikationen bei 3\% und Rerupturen bei 2\% dar. Komplikationen waren häufiger bei Verzögerung der Operation von mehr als 28 Tagen nach Unfalldatum [53].

Auch Bisson berichtet in seiner retrospektiven Auswertung von 45 mit Double-Inzision behandelten Patienten nach einem Follow-up von 6 Monaten über eine Verdopplung der Komplikationsrate von 20 auf $40 \%$ bei Operationen mehr als 15 Tage nach Verletzung. Dieser Unterschied war jedoch ohne Signifikanz. Es bestanden 11\% Neurapraxien des N. cutaneus antebrachii lateralis, $7 \%$ radioulnare Synostosen, 4\% mit Bewegungseinschränkung und $2 \%$ Rerupturen [42].

Beks et al. untersuchten mögliche Faktoren, die mit dem Auftreten von Komplikationen nach distaler Bizepssehnenrekonstruktion assoziiert sind. Von 373 Patienten, operiert von 2002 bis 2015, hatten 22\% eine Komplikation, und 5,3\% waren relevante Komplikationen. Im Einklang mit anderen Autoren war die Hauptkomplikation die Neurapraxie des N. cutaneus antebrachii lateralis. Die ventrale Single-Inzisions-Technik und Adipositas waren mit signifikant höheren Komplikationsraten verbunden. $18 \%$ der Patienten mit Komplikationen und $4 \%$ aller Patienten benötigten einen 2. operativen Eingriff [54].

\section{Zukunft: endoskopische Techniken?}

Aus den dargestellten Schwierigkeiten, von ventral den anatomischen Ansatz der Bizepssehne am ulnarseitigen posterioren Bereich der Tuberositas zu rekonstruieren und möglichst minimalinvasiv vorzugehen, um Komplikationen wie Neurapraxien und heterotope Ossifikationen zu vermeiden, werden endoskopische Techniken propagiert.

Gregory et al. publizierten erste Ergebnisse einer endoskopisch assistierten Rekonstruktion mittels eines Knochenankers. Die Studie umfasste 23 Patienten mit einem durchschnittlichen Follow-up von 26 Monaten. 25 Patienten waren zufrieden und der Verlauf ohne Komplikationen. Zwei Patienten hatten asymptomatische heterotope Ossifikationen. Ein Patient hatte einen neurografisch nachgewiesenen postoperativen Schaden des N. medianus und eine Ellbogensteife. Trotz operativer Neurolyse bestanden im Verlauf Sensibilitätsstörungen [55].

Bei einer von Phadnis et al. beschriebenen Technik werden nach kleiner transversaler Inzision von ventral in voller Supination, endoskopisch assistiert, nach Anfrischen des Footprints vom radialen Aspekt der Tuberositas 2 (proximal und distal) bikortikale Bohrungen in Richtung posteroulnar angelegt.

Cave

Beim Anlegen des Bohrkanals ist bei bikortikaler Bohrung eine Verletzung des N. interosseus posterior möglich.

Die Sehne wird geborgen, ausgerichtet und sowohl radialseitig als auch ulnarseitig mittels Fiberwire armiert. Durch die Bohrungen werden Kanülen vorgelegt, um jeweils Faden-Loops einzubringen, über die dann die Fibertape-Fäden der armierten Sehne von posteroulnar nach radioventral geshuttelt werden. Die an der radialen Seite der Sehne ausgeleiteten Fäden werden in das distale Bohrloch geführt und mittels Endobutton ventroradial fixiert ( $\triangleright$ Abb. 5e). Hierbei wird der Unterarm in Neutralposition gehalten und Traktion auf den distalen Faden gebracht, während der proximale Faden verknotet wird, um die Sehne auf den Footprint zu pressen.

Phadnis beschreibt bei bisher 28 behandelten Patienten eine störende heterotope Ossifikation als Komplikation. Während der Revision dieser Patienten konnte eine komplett am Footprint angeheilte distale Bizepssehne festgestellt werden [46].

Eine von anderen Autoren befürchtete Gefährdung des $\mathrm{R}$. profundus des $\mathrm{N}$. radialis konnte nicht nachgewiesen werden. In anatomischen Studien wurde eine durchschnittliche Distanz des Nervs vom posteroulnaren Bohrloch von $14 \mathrm{~mm}$ angegeben [56].

\section{Partialläsionen}

Partialrupturen der distalen Bizepssehne können traumatischer, aber auch oft degenerativer Natur sein. Schmerzen in der Antekubitalregion sind oft weniger ausgeprägt 
als bei Totalrupturen. Die klinisch-inspektorische und palpatorische Diagnose, inklusive des Hook-Tests, ist aufgrund der teils erhaltenen Insertion der Sehne oft weniger aussagekräftig als bei kompletten Läsionen. Mittels Sonografie und vor allem der MRT-Diagnostik lassen sich die Verletzungen jedoch gut darstellen [18].

\section{Therapeutisches Vorgehen}

Der erste Behandlungsansatz ist konservativ mit Aktivitätsmodifikation und entzündungshemmender Medikation. Bei Leistungssportlern, vorwiegend Ruderern und Kletterern, zeigt in unserer Erfahrung die sonografisch kontrollierte Infiltration mit PRP positive Resultate bei chronischer Tendinitis der distalen Bizepssehne ohne strukturell-morphologische Schäden der Sehne im MRT.

Auch wenn die Evidenzlage für diese Therapie sehr schwach ist, erachten wir in ausgewählten Fällen die Eigenblutinfiltration als eines der Werkzeuge zur Behandlung der distalen Bizepstendinopathie. Bei Persistenz der Beschwerden oder subtotalen Partialrupturen mit $>50 \%$ Beteiligung der Sehne wird ein chirurgisches Débridement der degenerativen Sehne mit anatomischem Repair empfohlen [32,33]. Ein endoskopischer Therapieansatz erscheint hierfür aufgrund des propagierten, weichteilschonenden Vorgehens attraktiv, hat sich jedoch im klinischen Alltag bis heute nicht durchgesetzt.

\section{Chronische Läsionen}

Ab 4 Wochen posttraumatisch werden Rupturen als chronisch eingestuft [57]. In chronischen Fällen ist der Muskel meist retrahiert und die Bizepssehne verkürzt und degenerativ verändert. Neben dem Versuch des direkten Repairs steht die biologische Augmentation mittels Graft-Rekonstruktion zur Verfügung ( $\triangleright$ Abb. 6). Verwendung finden Semitendinosus-, Flexor-carpi-radialis-, Fascia-lata- und Achillessehnen-Grafts.

Morrey et al. untersuchten retrospektiv 188 Double-Inzisions-Rekonstruktionen. Sie identifizierten Patienten, bei denen eine Refixation lediglich in $>60^{\circ}$ Flexion möglich war (19 chronische und 4 akute), und verglichen die Ergebnisse mit einer Matched-Pair-Kontrollgruppe mit Rekonstruktion in $<30^{\circ}$ Flexion. Bezüglich Mayo Elbow Score, Bewegungsumfang, subjektiver Zufriedenheit, Kraft, Komplikationen und Arbeitswiederaufnahme bestand kein signifikanter Unterschied.

Bei den in hoher Flexion rekonstruierten Patienten kam es zu 3 vorübergehenden Neurapraxien des N. cutaneus antebrachii lateralis und einer traumatischen Reruptur. Kontrakturen, Synostosen oder persistierende neurologische Defizite wurden nicht beobachtet. In der Kontrollgruppe kam es zu 4 transienten neurologischen Komplikationen (3 N.-radialis-Dysfunktionen und 1 N.-cutaneus-antebrachii-lateralis-Neurapraxie). Die Autoren schlussfolgerten, dass die Sehne bei bis zu $90^{\circ}$ Flexions- stellung verlässlich refixiert werden kann und ein deutliches Stretching des verkürzten Muskels möglich sei. Zur Entscheidungsfindung, ob ein Allograft verwendet wird oder nicht, sollte eher die Beurteilung der Sehnenqualität herangezogen werden [57].

Wiley et al. verglichen das Ergebnis zweier Patientengruppen, bestehend aus jeweils 7 Patienten mit chronischen Rupturen. Eine Gruppe war mittels Double-Incision-Semitendinosus-Autograft behandelt worden, die andere konservativ. Das Follow-up betrug 30 Monate, und alle Patienten der Graftgruppe waren mit dem Ergebnis zufrieden. Es kam zu keiner Verletzung des N. radialis, Rerupturen oder heterotopen Ossifikationen.

Bei der konservativen Gruppe zeigte sich ein Defizit von $20 \%$ in Flexion und Supination. In Flexion war die Kraftreduktion verglichen mit Werten einer Kontrollgruppe signifikant [58].

Hallam et al. beschrieben für die Rekonstruktion mittels Semitendinosus-Graft ebenfalls gute Ergebnisse in einer Gruppe von 9 Patienten mit einem durchschnittlichen Mayo Elbow Score von 96,3 [45].

Levy et al. berichteten über 5 Patienten mit chronischer Ruptur und Versorgung mittels Flexor-carpi-radialis-Graft $\geq 3$ Monate nach dem Unfall. Alle Patienten konnten zu körperlicher und sportlicher Aktivität zurückkehren. Verglichen mit der Gegenseite bestanden 109\% Supinationskraft und 103\% Supinationsausdauer. In Flexion betrugen diese Werte 97 und 89\% [58].

\section{Distale Trizepssehnenruptur}

\section{Inzidenz und Ätiologie}

Distale Trizepssehnenrupturen machen lediglich $1 \%$ aller Sehnenrupturen aus $[59,60]$ und gehören somit zu den seltensten Verletzungen der oberen Extremität. 25\% der distalen Trizepssehnenrupturen sind Partialrupturen [61]. Die Partialrupturen sind am häufigsten medial lokalisiert [60]. Der häufigste Läsionsort für eine distale Trizepssehnenruptur ist die distale ossäre Insertion [62].

Distale Trizepssehnenrupturen sind bei Männern doppelt so oft wie bei Frauen diagnostiziert [59]. Relativ gesehen sind am häufigsten Sportler betroffen (insbesondere Kraftsportler, Gewichtheber und American-Football-Spieler). Absolut gesehen ist jedoch das Verletzungsbild am häufigsten bei körperlich arbeitenden Personen zwischen dem 40. und dem 50. Lebensjahr zu beobachten [63].

\section{Risikofaktoren}

Zu den Risikofaktoren für eine distale Trizepssehnenruptur zählen der chronische Gebrauch anaboler Steroide und Kortikosteroide. Auch nach repetitiven Infiltrationen 


\section{FALLBEISPIEL}

Das Fallbeispiel stellt die Semitendinosus-Graft-Rekonstruktion einer retrahierten, chronischen, linksseitigen distalen Bizepssehnenruptur dar ( $\bullet$ Abb. 6). Der intraoperative Situs zeigt die Semitendinosus-Graft-Rekonstruktion über eine Single-Inzision. Ein gutes postoperatives Bewegungsausmaß konnte so erreicht werden.
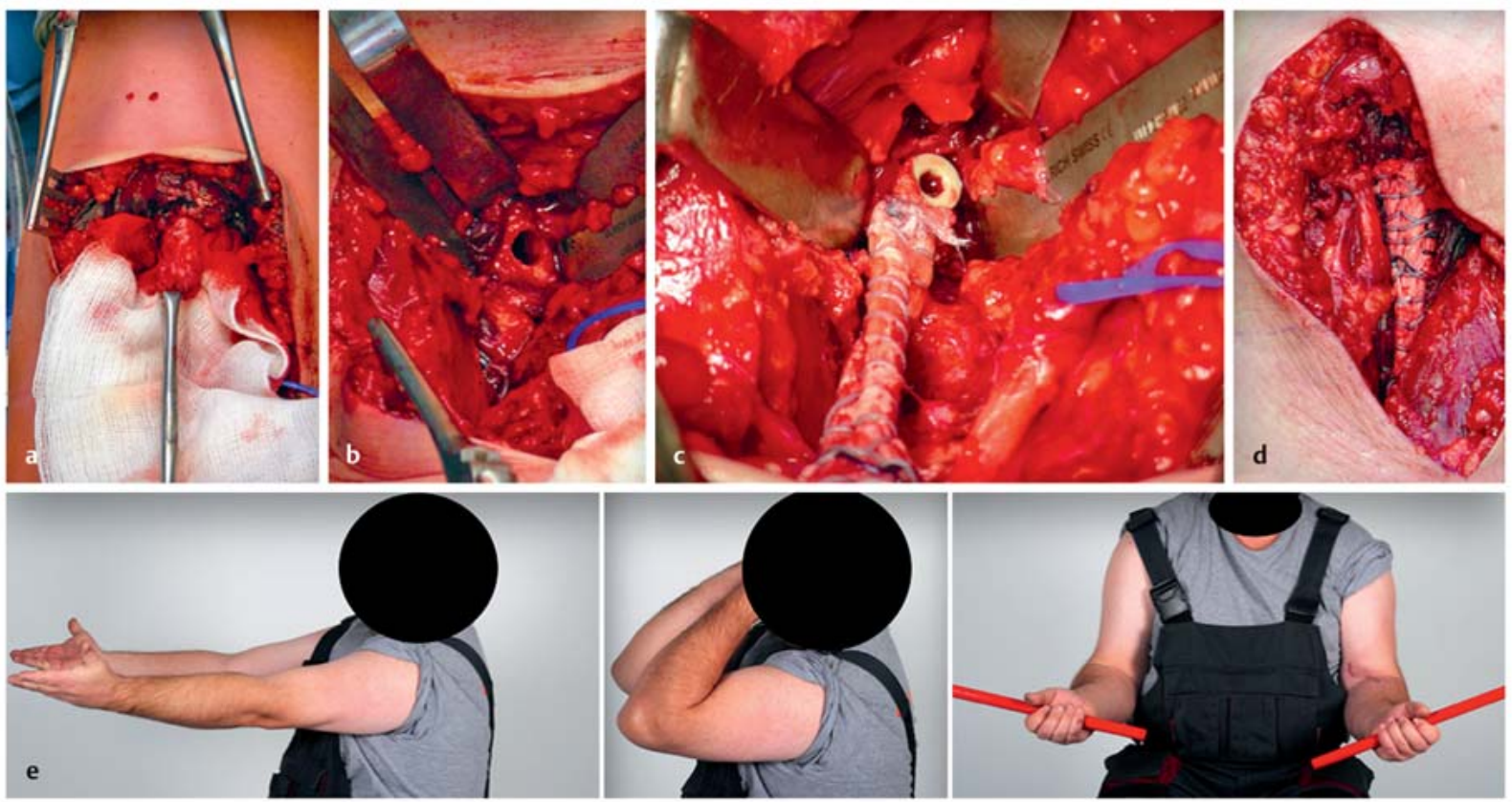

- Abb. 6 Semitendinosus-Graft-Rekonstruktion mit Single-Inzision. a Darstellung des retrahierten, degenerativ veränderten Sehnenstumpfes. b Bohrung an der Tuberositas radii für den Knochenanker. c Per Knochenanker fixiertes, mit dem Reststumpf der Sehne verflochtenes Semitendinosus-Graft. d Semitendinosus-Graft in situ. e Postoperatives Bewegungsausmaß des Patienten.

mit Kortison bei Tendinitis der distalen Trizepssehne oder bei Bursitis olecrani konnten vermehrt Rupturen der distalen Trizepssehne nachgewiesen werden. Des Weiteren können gewisse systemische Erkrankungen wie renale Osteodystrophie, Hyperparathyreoidismus oder Diabetes mellitus für eine distale Trizepssehnenruptur prädisponieren $[63,64]$.

\section{Anatomie}

Der M. triceps brachii fungiert als Hauptstrecker im Ellenbogengelenk. Er besteht aus 3 Muskelbäuchen (Caput longum, Caput laterale und Caput mediale) und setzt breitflächig etwa 12-14 mm distal der Olecranonspitze an [64]. Der Ansatzbereich (Footprint) der gesamten Sehne beträgt je nach Literatur 399-646 mm² $[64,65]$. Die Sehne ist aufgeteilt in einen medialen Anteil, der direkt zum Olecranon zieht, und einen lateralen Anteil, welcher breitflächig (lateral Triceps Expansion) mit einem angulierten Faserverlauf in die Faszie des M. extensor carpi ulnaris und in die tiefe Faszie des M. anconeus einstrahlt [59]. Dieser angulierte Faserverlauf ist verantwortlich dafür, dass die distale Trizepssehne häufig nicht vollständig rupturiert und retrahiert.
Die Innervation erfolgt über den N. radialis (C6-C8). Bei einer operativen Versorgung sind gewisse, neurovaskuläre Strukturen zu beachten. Der N. cutaneus brachii lateralis inferior zieht über den distalen, dorsalen Oberarm bis zum Olecranon, der N. cutaneus antebrachii posterior verläuft über den Epicondylus radialis humeri zum Olecranon. Beides sind Nervenäste des N. radialis.

Die A. collateralis media zieht über den distalen Humerus dorsal, die A. interossea recurrens verläuft radial des Olecranon und die A. collateralis ulnaris superior ulnar des Olecranon.

\section{Diagnostik}

\section{Anamnese}

Es gibt verschiedene Verletzungsmechanismen, die zu einer distalen Trizepssehnenruptur führen können. Am häufigsten kommt eine Ruptur bei kraftvoller, exzentrischer Belastung auf einen sich kontrahierenden M. triceps brachii vor, etwa bei einem Sturz auf den ausgestreckten Arm oder während des Krafttrainings oder Gewichthebens. 
Eine distale Trizepssehnenruptur durch direktes Trauma ist eher selten [63]. Die Patienten beschreiben häufig einen schmerzhaften Knall oder ein schmerzhaftes Gefühl des Reißens.

\section{Klinische Untersuchung}

\section{Merke}

Häufig kann eine distale Trizepssehnenruptur bereits mittels klinischer Untersuchung diagnostiziert werden.

Meist lässt sich eine Delle proximal des Olecranon und eine schmerzhafte und geschwollene Bursa olecrani palpieren. Des Weiteren beklagen die Patienten ein subjektives Extensionsdefizit, und es zeigt sich eine Schwäche bei Extension gegen Widerstand. Die Unfähigkeit, den Arm im Ellenbogengelenk gegen die Schwerkraft zu strecken, spricht meist für eine Komplettruptur der distalen Trizepssehne. Dennoch wird die Hälfte der akuten, distalen Trizepssehnenrupturen initial übersehen (die durchschnittliche Zeitspanne zwischen der Verletzung und der operativen Versorgung beträgt 24 Tage [66]), da der laterale Trizepssehnenanteil häufig bereits für eine Extension gegen die Schwerkraft ausreicht [62].

Analog zur Achillessehne kann man in der klinischen Untersuchung einen modifizierten Thompson-Test durchführen ( $\triangleright$ Abb. 7). Hierfür komprimiert der Untersucher kräftig mit einer Hand den M. triceps brachii in transversaler Richtung. Bei ausbleibender Extension im Ellenbogengelenk spricht dies meist für eine vollständige Ruptur [59]. Des Weiteren ist die Unfähigkeit, den Ellenbogen in Extension zu halten, auch Triceps Lag Sign genannt, ein weiterer Hinweis für eine Ruptur.

\section{Bildgebung}

Zur bildgebenden Diagnostik gehört immer ein konventionelles Röntgen in 2 Ebenen, insbesondere zum Ausschluss relevanter Frakturen. In einem Notfall kann eine relevante, distale Trizepssehnenruptur, wenn ein MRT nicht zur Verfügung steht, mittels Sonografie diagnostiziert werden. Zur Differenzierung einer Total- versus Partialruptur und auch zur Evaluation der Retraktion der distalen Trizepssehne ist eine MRT-Untersuchung die beste Modalität. Die MRT-Untersuchung sollte, um die Dehiszenz adäquat beurteilen zu können, in Flexionsstellung erfolgen. Bei knöchernen Ausrissen kann zur operativen Planung auch ein CT durchgeführt werden ( $\triangleright$ Abb. 8).

\section{Therapie}

Konservative Therapie

Bezüglich des Outcomes der konservativen Therapie gibt es bis dato nur wenig Literatur. Die Indikation für eine konservative Therapie sind:

- Partialrupturen der distalen Trizepssehne,

- niedriger, funktioneller Anspruch und

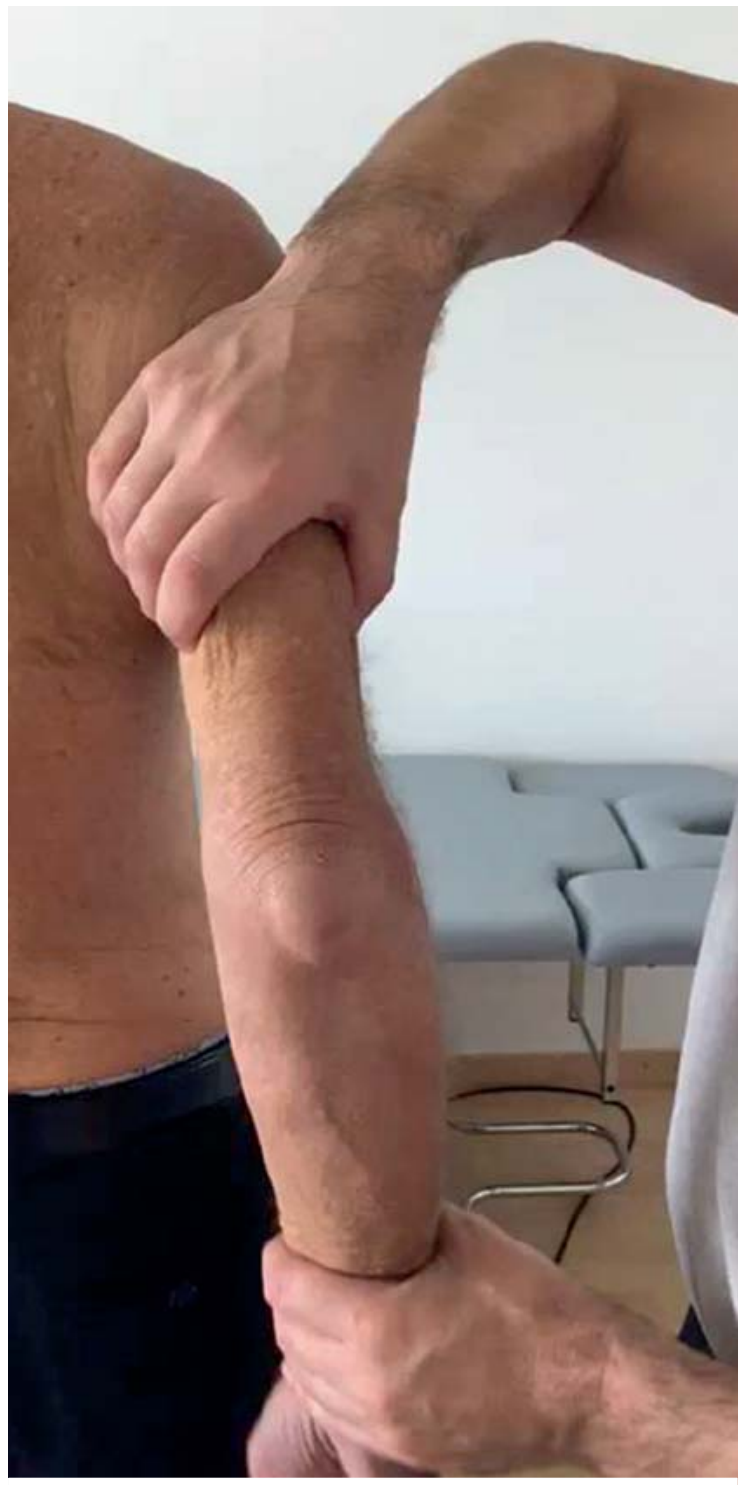

- Abb. 7 Modifizierter Thompson-Test.

- relevante Komorbiditäten, die eine Einheilung der Sehne nach einer operativen Versorgung kompromittieren würden.

Bei konservativer Therapie wird eine kurze Ruhigstellung in $30^{\circ}$ Flexion für 3-4 Wochen mit anschließend vorsichtiger Steigerung des Bewegungsumfangs und der Belastung unter physiotherapeutischer Anleitung empfohlen. Die adjuvante, medikamentöse Therapie mit NSAID und Infiltrationstherapie mit PRP ist umstritten [61]. Mair et al. [60] haben in ihrer Studie 10 American-Football-Spieler mit einer Partialruptur untersucht, wobei 6 ohne operative Versorgung ohne funktionelles Defizit geheilt sind (die Patienten erreichten ihr ursprüngliches Sportniveau). 

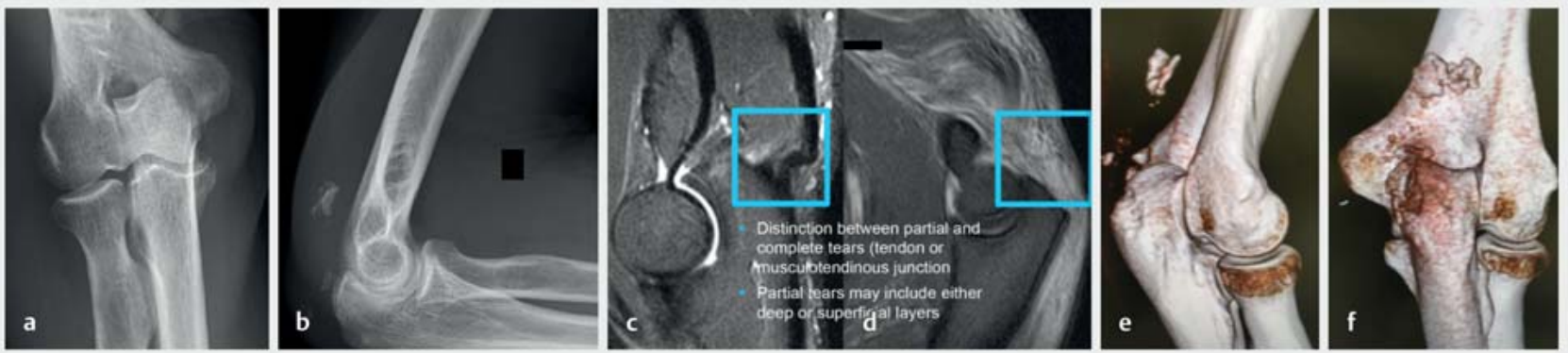

- Abb. 8 Bildgebende Diagnostik bei V.a. Trizepssehnenruptur. a Konventionelle Röntgenuntersuchung in 2 Ebenen, die einen ossären Ausriss der distalen Trizepssehne zeigt (hier: Strahlengang a.-p.). b Seitlicher Strahlengang. c Differenzierung zwischen partiellem und komlettem Sehnenriss (Sehne oder muskulotendinöser Übergang). Partialrupturen können entweder tiefe oder oberflächliche Anteile betreffen. $\mathbf{d}$ Totalruptur der Trizepssehne im MRT. e CT zur Frakturbilanzierung bei ossärem Ausriss der distalen Trizepssehne, Sicht von lateral. f Sicht von posterior

\section{Merke}

Dies zeigt, dass Partialrupturen auch bei Patienten mit hohem funktionellem Anspruch konservativ behandelt werden können, obwohl die meisten distalen Trizepssehnenrupturen operativ behandelt werden.

\section{Operative Therapie}

Die Indikationen für eine operative Versorgung distaler Trizepssehnenrupturen sind:

- Partialrupturen bei Patienten mit hohem funktionellem Anspruch (z. B. Kraftverlust),

- Partialrupturen, bei welchen die konservative Therapie fehlgeschlagen ist, und

- Komplettrupturen.

Wenn man sich für eine operative Versorgung entscheidet, sollte diese in den ersten 2-3 Wochen durchgeführt werden. Hierfür kommen verschiedene operative Techniken mit dem Ziel einer anatomischen und flächigen Rekonstruktion in Frage. Einerseits besteht die Möglichkeit, die Sehne mittels transossären Nähten zu refixieren, anderseits kann dies mit Ankern erfolgen. Als 3. Variante kann eine Hybridtechnik im Sinne einer Kombination von transossären Nähten mit Ankern gewählt werden.

Die operative Versorgung kann in Bauch- oder Seitenlage durchgeführt werden. Eine Blutsperre kann einerseits hilfreich sein, jedoch bei retrahierter, distaler Trizepssehne auch die Mobilisation und Reposition erschweren. Der posteriore Zugang sollte so weit nach distal gezogen werden, bis die vollständige Insertion der distalen Trizepssehne einsehbar ist.

Partialrupturen lassen sich gut arthroskopisch versorgen ( Abb.9), insbesondere Partialläsionen des medialen Anteils und degenerative, bursaseitige Partialläsionen der distalen Trizepssehne. In der Literatur haben sich bis dato jedoch nur wenige Studien mit arthroskopischen Operationsverfahren auseinandergesetzt.
Bei subtotalen und kompletten Rupturen der distalen Trizepssehne werden meist offene Verfahren angewendet. Hierfür gibt es, wie bereits erwähnt, verschiedene operative Techniken. Man kann dies mittels klassischer, transossärer Nähte, Double-Row-Technik in geknoteter Technik oder mit knotenfreien Ankern, Double-Row-Technik mit All-Suture-Ankern oder Hybridtechnik angehen [65, 66] (- Abb. 10).

\section{Ergebnisse}

Biomechanische Resultate: Es gibt verschiedene biomechanische Studien, welche die Stabilität der verschiedenen, operativen Techniken untersucht haben. Carpenter et al. [67] konnten keine relevanten Unterschiede bezüglich der Stabilität bei transossären Nähten gegenüber einem Repair mit Ankern zeigen, vielmehr scheinen die Anzahl der Fäden bzw. Ankerreihen eine entscheidende Rolle zu spielen. Bei der Platzierung einer 2. Ankerreihe sollte man zur Vermeidung einer Perforation nach intraartikulär jedoch Vorsicht walten lassen.

Scheiderer et al. [65] hingegen konnten in ihrer Studie zeigen, dass eine V-förmige Rekonstruktion in Hybridtechnik mit einem unikortikalen Button bezüglich Dislokation der refixierten Sehne einer Refixation mittels transossären Nähten allein signifikant überlegen ist. Auch bezüglich Belastbarkeit schnitt die Hybridtechnik gegenüber einer Refixation mittels transossärer Nähte allein oder Refixation mittels knotenfreier Suture-Bridge-Technik besser ab.

Klinische Resultate: In den klinischen Verlaufskontrollen zeigte sich bei unterschiedlichen Operationstechniken bezüglich Zufriedenheit der Patienten keine Korrelation. In einer von Mirzayan et al. [63] durchgeführten Studie konnten jedoch bei Patienten mit transossären Nähten häufiger Rerupturen, häufiger Reinterventionen und damit einhergehend längere Hospitalisationen beobachtet werden. Dunn et al. [66] konnten in ihrem Review zeigen, 

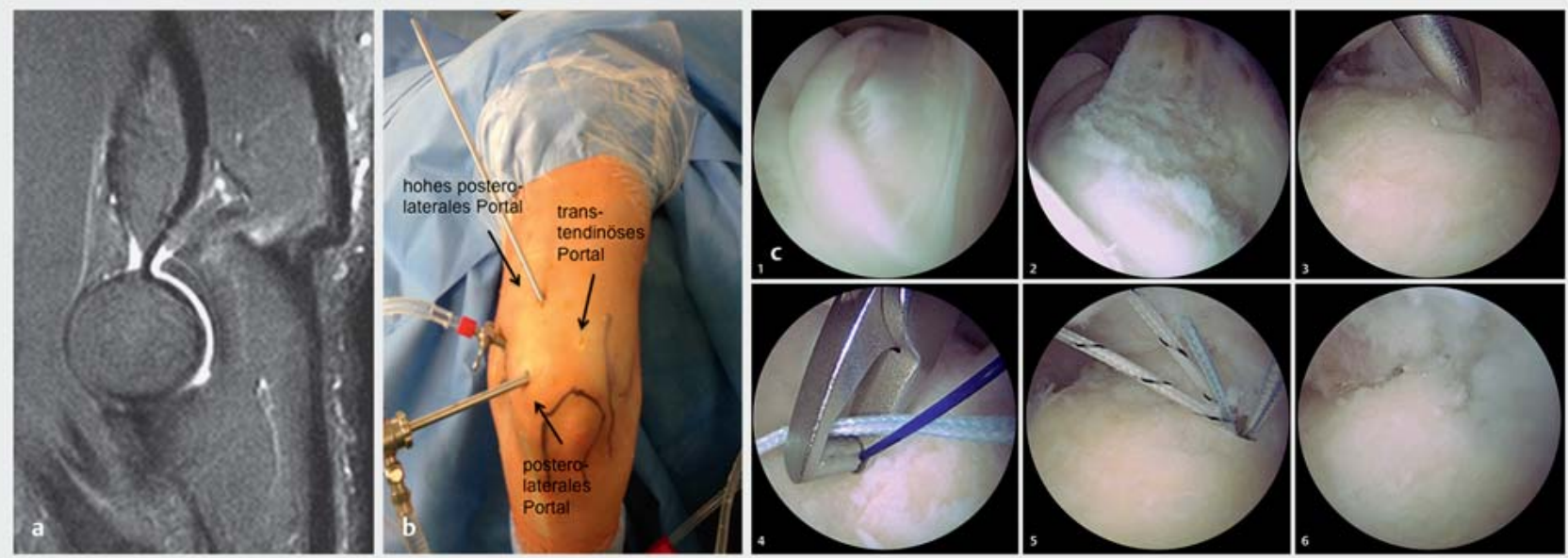

- Abb. 9 Partialruptur der distalen Trizepssehne (Quelle: Gerhardt C, Scheibel M. Trizepsrupturen. In: Müller L, Hollinger B, Burkhart K, Hrsg. Expertise Ellenbogen. 1. Auflage. Stuttgart: Thieme; 2016. doi:10.1055/b-003-128229). a Nachweis im MRT. b Zugänge für das arthroskopisches Verfahren in Seitenlage. c Intraoperative Bilder bei arthroskopischem Verfahren bei Ruptur des medialen Anteiles des distalen Trizepssehne. c1 Intraoperativer Situs. c2 Nach Debridement und Anfrischen des Footprints. c3 Pfriem für Ankerplatzierung. c4 Durchshutteln der FiberwireFäden mittels PDS-Faden. c5 In Matratzen-Technik durchgezogene Fäden. c6 Sehnenrekonstruktion nach Abknoten der Fäden.
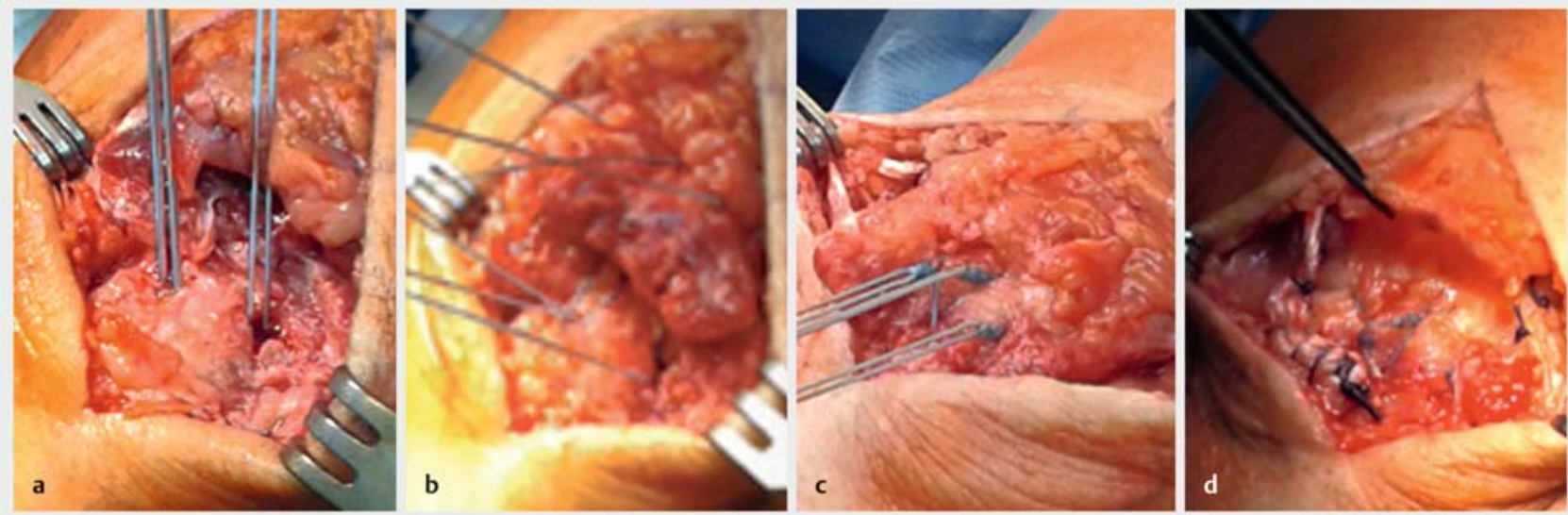

- Abb. 10 Fixationstechnik bei distaler Trizepssehnenrekonstruktion, hier mittels All-suture Ankern in Double-Row-Technik mit Feinadaptation. a Proximale Anker mit Fäden (Quelle: Gerhardt C, Scheibel M. Therapeutisches Vorgehen. In: Müller L, Hollinger B, Burkhart K, Hrsg. Expertise Ellenbogen. 1. Auflage. Stuttgart: Thieme; 2016. doi:10.1055/b-003-128229). b Einbringen der Fäden durch die Sehne (Quelle: Gerhardt C, Scheibel M. Therapeutisches Vorgehen. In: Müller L, Hollinger B, Burkhart K, Hrsg. Expertise Ellenbogen. 1. Auflage. Stuttgart: Thieme; 2016. doi:10.1055/b-003-128229). c Geknotete proximale Reihe. d In distaler Ankerreihe abgespannte Fäden und Feinadaptation (Quelle: Gerhardt C, Scheibel M. Therapeutisches Vorgehen. In: Müller L, Hollinger B, Burkhart K, Hrsg. Expertise Ellenbogen. 1. Auflage. Stuttgart: Thieme; 2016. doi:10.1055/b-003-128229). 
dass 89\% aller operierten Patienten ihr Aktivitätslevel wieder erreichen konnten.

Eine einheitliche und standardisierte Evaluation des Outcomes ist jedoch bei fehlenden Scores nicht möglich.

\section{Postoperative Rehabilitation}

Die postoperative Behandlung ist von der angewandten Operationstechnik abhängig. Generell kann jedoch folgendes Nachbehandlungsschema empfohlen werden [68]:

- Woche 1 und 2: Ruhigstellung in einer Oberarmgipsschiene oder äquivalent in $30-45^{\circ}$ Flexion.

- Woche 3 und 4: Bewegungsschiene mit Flexionseinschränkung von $90^{\circ}$, aktive Supination und Pronation erlaubt.

- Woche 5 und 6: Bewegungsschiene mit Flexionseinschränkung von maximal $120^{\circ}$.

- Woche 7-12: freier Bewegungsumfang ohne Schiene, isometrische Extension im Ellenbogengelenk.

\section{Komplikationen}

In der durch Dunn et al. [66] durchgeführten Metaanalyse waren die häufigsten Komplikationen Rerupturen (3-13\%), Infekte (0-5,2\%), Einschränkung des Bewegungsumfangs (9-25\%) und Lähmung des N. radialis (6\%). Keener et al. [64] kamen in ihrer Studie zu ähnlichen Resultaten. Am häufigsten waren ebenfalls Rerupturen (bis 20\%), Olecranon-Avulsionsfrakturen, reduzierter Bewegungsumfang, Lähmung des N. radialis und Neuropathie des N. ulnaris. Auch konnten sie zeigen, dass eine operative Versorgung in den ersten 2-3 Wochen erfolgversprechender ist.

\section{Chronische Rupturen}

Chronische Rupturen der distalen Trizepssehne sind definiert als Rupturen, die mehr als 6 Wochen alt sind. Bei chronischen Rupturen stehen Schmerzen und Kraftverlust im Vordergrund. Zur operativen Versorgung sind multiple Verfahren wie Anconeusinterponat, Achillessehnenrotationslappen sowie M. plantaris longus oder Hamstrings-Augmentation beschrieben. Die Resultate sind heterogen und sowohl abhängig von der Retraktion und Qualität der Sehne als auch von den Komorbiditäten $[59,64]$.

\section{KERNAUSSAGEN}

- Die Ruptur der distalen Bizeps- und Trizepssehne kann oft klinisch diagnostiziert werden und tritt hauptsächlich bei körperlich tätigen Männern und Kraftsportlern auf.

- Distale Trizepssehnenrupturen sind ein seltenes Verletzungsbild und werden häufig verpasst, daher ist eine sorgfältige klinische Untersuchung unabdingbar.

- Goldstandard für die Bildgebung distaler Bizeps- und Trizepssehnenrupturen ist das MRT

- In der Regel wird eine operative Versorgung empfohlen. Zur Gewährleistung eines guten Outcomes sollte diese in den ersten 2-3 Wochen erfolgen.

- Konservatives Management ist eine Option bei Partialrupturen und bei Patienten mit geringem funktionellem Anspruch und/ oder höherem Alter.

- Die Ultraschall- und MRT Untersuchung ist besonders hilfreich bei Partialrupturen und Rerupturen.

- Rekonstruktionen der distalen Bizepssehne in Single- und DoubleInzisions-Technik ergeben unabhängig von der Fixationstechnik vergleichbar gute Ergebnisse.

- Die klinische Relevanz der anatomischen Rekonstruktion der distalen Bizepssehne muss noch definiert werden.

- Minor-Komplikationen bei distaler Bizepssehnenrefixation sind häufig und betreffen vor allem den N. cutaneus antebrachii lateralis in Form von transienten Neurapraxien.

- Eine Blutsperre kann eine Reposition der Sehne erschweren.

- Chronische und retrahierte Rupturen, welche mit einem Interponat operativ versorgt werden, weisen schlechtere Ergebnisse auf als direkt refixierte Sehnen, jedoch bessere Resultate als konservativ behandelte Rupturen.

- Ob die vielversprechenden Ansätze endoskopischer Techniken für eine komplikationsärmere und anatomische Rekonstruktion der Standard der Zukunft sind, wird sich zeigen.

Interessenkonflikt

Erklärung zu finanziellen Interessen

Forschungsförderung erhalten: nein; Honorar/geldwerten Vorteil für Referententätigkeit erhalten: nein; Bezahlter Berater/interner Schulungsreferent/Gehaltsempfänger: ja; Patent/Geschäftsanteile/Aktien (Autor/Partner, Ehepartner, Kinder) an Firma (Nicht-Sponsor der Veranstaltung): nein; Patent/Geschäftsanteile/Aktien (Autor/Partner, Ehepartner, Kinder) an Firma (Sponsor der Veranstaltung): nein.

Erklärung zu nichtfinanziellen Interessen

Die Autorinnen/Autoren geben an, dass kein Interessenkonflikt besteht. 


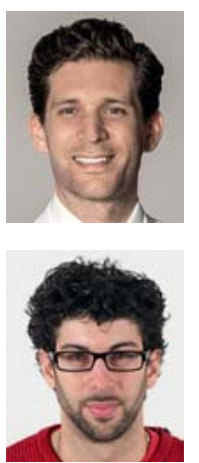

\section{Florian Freislederer}

Oberarzt, Schulter- und Ellbogenchirurgie, Schulthess Klinik Zürich, Schweiz

\section{Donato Papillo}

Dr. med., Assistenzarzt, Orthopädische Chirurgie und Traumatologie des Bewegungsapparates, Kantonsspital Frauenfeld

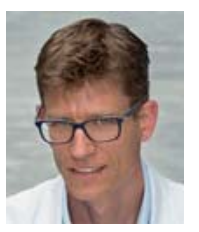

\section{Michael Glanzmann}

Dr. med., Leitender Arzt, Schulter- und Ellbogenchirurgie Schulthess Klinik, Zürich, Schweiz

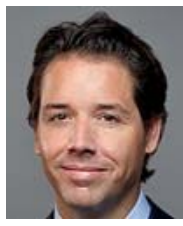

\section{Markus Scheibel}

Prof. Dr. med., Chefarzt Schulter- und Ellbogenchirurgie Schulthess Klinik, Zürich

\section{Wissenschaftlich verantwortlich} gemäß Zertifizierungsbestimmungen

Wissenschaftlich verantwortlich gemäß Zertifizierungsbestimmungen für diesen Beitrag ist Prof. Dr. med. Markus Scheibel, Zürich.

\section{Korrespondenzadresse}

Prof. Dr. med. Markus Scheibel

Schulthess Klinik

Lengghalde 2

8008 Zürich, Schweiz

Markus.Scheibel@kws.ch

\section{Literatur}

[1] Safran MR, Grahan SM. Distal biceps tendon ruptures: incidence, demographics and the effect of smoking. Clin Orthop Relat Res 2002; (404): 275-283

[2] Kelly MP, Perkinson SG, Ablove RH et al. Distal biceps tendon ruptures: an epidemiological analysis using a large population database. Am J Sports Med 2015; 43: 2012-2017. doi:10.1177/0363546515587738

[3] Ramsey ML. Distal biceps tendon injuries: diagnosis and management. J Am Acad Orthop Surg 1999; 7: 199-207

[4] D'Alessandro DF, Shields CL jr., Tibone JE et al. Repair of distal biceps tendon ruptures in athletes. Am J Sports Med 1993; 21 : 114-119. doi:10.1177/036354659302100119

[5] Miyamoto RG, Elser F, Millett PJ. Distal biceps tendon injuries. J Bone Joint Surg Am 2010; 92: 2128-2138. doi:10.2106/JBJS. 1.01213
[6] Visuri T, Lindholm H. Bilateral distal biceps tendon avulsions with use of anabolic steroids. Official J Am Coll Sports Med 1994: 941-944

[7] Kanayama G, DeLuca J, Meehan WP 3rd et al. Ruptured tendons in anabolic-androgenic steroid users: a cross-sectional cohort study. Am J Sports Med 2015; 43: 2638-2644. doi:10.1177/0363546515602010

[8] Seiler JG 3rd, Parker LM, Chamberland PD et al. The distal biceps tendon. Two potential mechanism involved in its rupture: Arterial supply and mechanical impingement. J Shoulder Elbow Surg 1995; 4: 149-156

[9] Stevens K, Kwak A, Poplawski S. The biceps muscle from shoulder to elbow. Semin Musculoskelet Radiol 2012; 16: 296-315. doi:10.1055/s-0032-1327004

[10] Eames MH, Bain GI, Fogg QA et al. Distal biceps tendon anatomy: a cadaveric study. J Bone Joint Surg Am 2007; 89: 10441049. doi:10.2106/JBJS.D.02992

[11] Jarrett CD, Brown BT, Schmidt CC. Anatomical distal biceps and triceps tendon repairs. Oper Tech Orthop 2013; 23: 215223. doi:10.1053/j.oto.2013.07.012

[12] Schmidt CC, Jarrett CD, Brown BT. The distal biceps tendon. J Hand Surg Am 2013; 38: 811-821; quiz 821. doi:10.1016/j. jhsa.2013.01.042

[13] O'Driscoll SW, Goncalves LB, Dietz P. The hook test for distal biceps tendon avulsion. Am J Sports Med 2007; 35: 18651869. doi: $10.1177 / 0363546507305016$

[14] Ruland RT. The biceps squeeze test for diagnosis of distal biceps tendon ruptures. Clin Orthop Relat Res 2005; 437: 128 131

[15] ElMaraghy A, Devereaux M, Tsoi K. The biceps crease interval for diagnosing complete distal biceps tendon ruptures. Clin Orthop Relat Res 2008; 466: 2255-2262. doi:10.1007| s11999-008-0334-0

[16] Metzman LS, Tivener KA. The supination-pronation test for distal biceps tendon rupture. Am J Orthop 2015; 44, 10: 361364

[17] Tagliafico A, Michaud J, Capaccio E et al. Ultrasound Demonstration of Distal Biceps Tendon Bifurcation: Normal and Abnormal Findings. Eur Radiol 2010; 20: 202-208

[18] Festa A, Mulieri PJ, Newman JS et al. Effectiveness of magnetic resonance imaging in detecting partial and complete distal biceps tendon rupture. J Hand Surg Am 2010; 35: 77-83. doi:10.1016/j.jhsa.2009.08.016

[19] Giuffrè BM, Moss MJ. Optimal positioning for MRI of the distal biceps brachii tendon: flexed abducted supinated view. AJR Am J Roentgenol 2004; 182: 944-946

[20] Vandenberghe M, van Riet R. Distal biceps ruptures: open and endoscopic techniques. Curr Rev Muskuloskelet Med 2016; 9: 215-223

[21] Freeman CR, McCormick KR, Mahoney D et al. Nonoperative treatment of distal biceps tendon ruptures compared with a historical control group. J Bone Joint Surg Am 2009; 91: 2329-2334. doi:10.2106/JBJS.H.01150

[22] Nesterenko S, Domire Z], Morrey BF et al. Elbow strength and endurance in patients with a ruptured distal biceps tendon. J Shoulder Elbow Surg 2010; 19: 184-189. doi:10.1016/j. jse.2009.06.001

[23] Rantanen J, Orava S. Rupture of the distal biceps tendon. A report of 19 patients treated with anatomic reinsertion, and a meta-analysis of 147 cases found in the literature. Am J Sports Med 1999; 27: 128-132 
[24] Chavan PR, Duquin TR, Bisson LJ. Repair of the ruptured distal biceps tendon: a systematic review. Am J Sports Med 2008; 36: 1618-1624. doi:10.1177/0363546508321482

[25] Kettler M, Tingart Lunger et al. Reattachment of distal tendon of biceps - Factors affecting the failure strength of the repair. J Bone Joint Surg 2008; 90: 103-106

[26] Mazzocca AD, Burton KJ, Romeo AA et al. Biomechanical evaluation of 4 techniques of distal biceps brachii tendon repair. Am J Sports Med 2007; 35: 252-258

[27] Berlet GC, Johnson JA, Milne AD. Distal biceps brachii tendon repair. An in vitro biomechanicl study of tendon reattachment. Am J Sports Med 1998; 26: 428-432

[28] Bisson LJ, de Perio JG, Weber AE et al. Is it safe to perform aggressive rehabilitation after distal biceps tendon repair using the modified 2-incision approach? A biomechanical study. Am J Sports Med 2007; 35: 2045-2050. doi:10.1177| 0363546507305679

[29] Idler CS, Montgomery WH 3rd, Lindsey DP et al. Distal biceps tendon repair: a biomechanical comparison of intact tendon and 2 repair techniques. Am J Sports Med 2006; 34: 968974. doi: $10.1177 / 0363546505284185$

[30] Lemos SE, Ebramzedeh E, Kvitne RS. A new technique: in vitro suture anchor fixation has superior yield strength to bone tunnel fixation for distal biceps tendon repair. Am J Sports Med 2004; 32: 406-410. doi:10.1177/0363546503261720

[31] Spang JT, Weinhold PS, Karas SG. A biomechanical comparison of EndoButton versus suture anchor repair of distal biceps tendon injuries. J Shoulder Elbow Surg 2006; 15: 509-514. doi:10.1016/j.jse.2005.09.020

[32] Vardakas DG, Musgrave DS, Varitimidis SE et al. Partial rupture of the distal biceps tendon. J Shoulder Elbow Surg 2001; 10: 377-379. doi:10.1067/mse.2001.116518

[33] Bain Gl. Treatment of partial distal biceps tendon tears. Sports Med Arthrosc Rev 2008; 16: 154-161

[34] McKee MD, Hirji R, Schemitsch EH et al. Patient-oriented functional outcome after repair of distal biceps tendon ruptures using a single-incision technique. J Shoulder Elbow Surg 2005; 14: 302-306. doi:10.1016/j.jse.2004.09.007

[35] Mazzocca AD, Bicos RA, Romeo AA et al. Repair of distal biceps tendon ruptures using a combined anatomic interference screw and cortical button. Tech Shoulder Elbow Surg 2005; 6: 108-115

[36] Greenberg JA, Fernandez J], Wang T et al. EndoButton-assisted repair of distal biceps tendon ruptures. J Shoulder Elbow Surg 2003; 12: 484-490. doi:10.1016/s1058-2746(03)00173-3

[37] Schmidt CC, Brown BT, Williams BG et al. The importance of preserving the radial tuberosity during distal biceps repair. J Bone Joint Surg Am 2015; 97: 2014-2023. doi:10.2106/JBJS. N.01221

[38] John CK, Field LD, Weiss KS et al. Single-incision repair of acute distal biceps ruptures by use of suture anchors. J Shoulder Elbow Surg 2007; 16: 78-83. doi:10.1016/j.jse.2006.03.002

[39] Heinzelmann AD, Savoie FH 3rd, Ramsey JR et al. A combined technique for distal biceps repair using a soft tissue button and biotenodesis interference screw. Am J Sports Med 2009; 37: 989-994. doi:10.1177/0363546508330130

[40] Boyd HB, Anderson LD. A method for reinsertion of the distal biceps brachii tendon. J Bone Joint Surg Am Vol 1961; 43: 1041-1043

[41] Failla JM, Amadio MD, Morrey BF et al. Proximal radioulnar synostosis after repair of distal biceps brachii rupture by the twoincision technique. Clin Orthop Relat Res 1990; (253): 133136
[42] Bisson L, Moyer M, Lanighan K et al. Complications associated with repair of a distal biceps rupture using the modified twoincision technique. J Shoulder Elbow Surg 2008; 17: 67S-71S. doi:10.1016/j.jse.2007.04.008

[43] Weinstein DM, Ciccone WJ 2nd, Buckler MC et al. Elbow function after repair of the distal biceps brachii tendon with a twoincision approach. J Shoulder Elbow Surg 2008; 17: 82S-86S. doi:10.1016/j.jse.2007.07.006

[44] Karunakar MA, Cha P, Stern PJ. Distal biceps ruptures. A followup of Boyd and Anderson repair. Clin Orthop Relat Res 1999; 363: $100-107$

[45] Hasan SA, Cordell CL, Rauls RB et al. Two-incision versus oneincision repair for distal biceps tendon rupture: a cadaveric study. J Shoulder Elbow Surg 2012; 21: 935-941. doi:10.1016/j.jse.2011.04.027

[46] Phadnis J, Bain G. Endoscopic-assisted distal biceps footprint repair. Tech Hand Up Extrem Surg 2015; 19: 55-59

[47] Schmidt CC, Brown BT, Qvick LM et al. Factors that determine supination strength following distal biceps repair. J Shoulder Elbow Surg 2016; 25: e324-e325. doi:10.1016/j. jse.2016.07.049

[48] Cohen SB, Buckley PS, Neuman B et al. A functional analysis of distal biceps tendon repair: single-incision Endobutton technique vs. two-incision modified Boyd-Anderson technique. Phys Sportsmed 2016; 44: 59-62. doi:10.1080/ 00913847.2016.1129260

[49] Shields E, Olsen JR, Williams RB et al. Distal biceps brachii tendon repairs: a single-incision technique using a cortical button with interference screw versus a double-incision technique using suture fixation through bone tunnels. Am J Sports Med 2015; 43: 1072-1076. doi:10.1177/0363546515570465

[50] Grewal R, Athwal GS, MacDermid JC et al. Single versus double-incision technique for the repair of acute distal biceps tendon ruptures: a randomized clinical trial. J Bonejoint Surg Am 2012; 94: 1166-1174. doi:10.2106/JBJS.K.00436

[51] Hudson J, Dunphy TR, Butler RK et al. Indomethacin does not reduce heterotopic ossification in two-incision distal biceps repairs. Orthopc J Sports Med 2018; 6. doi:10.1177/ 2325967118s00152

[52] Garon MT, Greenberg JA. Complications of distal biceps repair. Orthop Clin North Am 2016; 47: 435-444. doi:10.1016/j. ocl.2015.10.003

[53] Cain RA, Nydick JA, Stein MI et al. Complications following distal biceps repair. J Hand Surg Am 2012; 37: 2112-2117. doi:10.1016/j.jhsa.2012.06.022

[54] Beks RB, Claessen FM, Oh LS et al. Factors associated with adverse events after distal biceps tendon repair or reconstruction. J Shoulder Elbow Surg 2016; 25: 1229-1234. doi:10.1016/j.jse.2016.02.032

[55] Gregory T, Roure P, Fontes D. Repair of distal biceps tendon rupture using a suture anchor: description of a new endoscopic procedure. Am J Sports Med 2009; 37: 506-511. doi:10.1177/0363546508326985

[56] Bain GI, Prem H, Heptinstall RJ et al. Repair of distal biceps tendon rupture: A new technique using the endobutton. J Shoulder Elbow Surg 2000; 9: 120-126. doi:10.1067| 2000.102581

[57] Morrey ME, Abdel MP, Sanchez-Sotelo J et al. Primary repair of retracted distal biceps tendon ruptures in extreme flexion. J Shoulder Elbow Surg 2014; 23: 679-685. doi:10.1016/j. jse.2013.12.030

[58] Levy H], Mashoof AA, Morgan D. Repair of chronic ruptures of distal biceps tendon using flexor carpi radialis tendon graft. Am J Sports Med 2000; 28: 538-540 
[59] Yeh PC, Dodds SD, Smart RL et al. Distal triceps rupture. ] Am Acad Orthop Surg 2010; 18: 10

[60] Mair SD, Isbell WM, Gill T] et al. Triceps tendon ruptures in professional football players. Am J Sports Med 2004; 32: 4. doi:10.1177/0095399703258707

[61] Cheatham SW, Kolber M], Salamh PA et al. Rehabilitation of a partially torn distal triceps tendon after platelet rich plasma injection: a case report. Int J Sports Physical Ther 2013; 8: 10

[62] van Riet RP, Morrey BF, Ho E et al. Surgical treatment of distal triceps ruptures. J Bone Joint Surg Am 2003; 85: 1961-1967

[63] Mirzayan R, Acevedo DC, Sodl JF et al. Operative management of acute triceps tendon ruptures: review of 184 cases. Am J Sports Med 2018; 46: 8. doi:10.1177/0363546518757426

[64] Keener JD, Sethi PM. Distal triceps tendon injuries. Hand Clin 2015; 31: 10. doi:10.1016/j.hcl.2015.06.010

[65] Scheiderer B, Imhoff FB, Morikawa D et al. The V-shaped distal triceps tendon repair: a comparative biomechanical analysis. Am J Sports Med 2018; 46: 6. doi:10.1177| 0363546518771359
[66] Dunn JC, Kusnezov N, Fares A et al. Triceps tendon ruptures: a systematic review. Hand (N Y) 2017; 12: 431-438. doi:10.1177/1558944716677338

[67] Carpenter SR, Stroh DA, Melvani R et al. Distal triceps transosseous cruciate versus suture anchor repair using equal constructs: a biomechanical comparison. J Shoulder Elbow Surg 2018; 27: 2052-2056. doi:10.1016/j.jse.2018.05.025

[68] Kocialkowski C, Carter R, Peach C. Triceps tendon rupture: repair and rehabilitation. Shoulder Elbow 2018; 10: 62-65

\section{Bibliografie}

DOI https://doi.org/10.1055/a-0999-8250

online publiziert 09.07 .2020 |Z Orthop Unfall 2020; 158: 663-682 @ Georg Thieme Verlag KG Stuttgart · New York ISSN 1864-6697 


\section{Punkte sammeln auf CME.thieme.de}

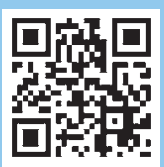

Diese Fortbildungseinheit ist in der Regel 12 Monate online für die Teilnahme verfügbar.

Den genauen Einsendeschluss finden Sie unter https://cme.thieme.de/.

Sollten Sie Fragen zur Online-Teilnahme haben, finden Sie unter https://cme.thieme.de/hilfe

eine ausführliche Anleitung. Wir wünschen viel Erfolg beim Beantworten

der Fragen!

Unter https://eref.thieme.de/CXDRF2A oder über den QR-Code kommen Sie

direkt zur Startseite des Wissenstests.

VNR 2760512020158725404

\section{Frage 1}

Welche Aussage trifft für die Biomechanik des distalen M. biceps brachii zu?

A Im Gegensatz zur Schulter ist die Funktion des M. biceps brachii am Ellenbogen von geringer Bedeutung.

B Die kurze Bizepssehne ist eher für Supinations- als Flexionsbewegungen verantwortlich.

C Die lange Bizepssehne setzt proximaler als die kurze Bizepssehne an.

D Der Lacertus fibrosus strahlt lateral in die Unterarmfaszie ein und inseriert am Radius.

E Die Integrität des Lacertus fibrosus erleichtert eine proximale Migration des Muskelbauches bei chronischen Rupturen.

\section{Frage 2}

Welche Aussage bezüglich traumatischer, distaler Bizepssehnenrupturen trifft zu?

A Ein Sturz auf den flektierten Arm oder abrupte Pronation sind typische Mechanismen.

B Im Vordergrund steht die schmerzhafte, teils auch schmerzlose Krafteinschränkung in Supination und Flexion.

C Die Bildung eines Hämatoms ist immer vorhanden.

D Ein hörbares Schnappen wird selten berichtet.

E Die Retraktion des Muskelbauches mit „Reverse-Popeye-Zeichen“" ist bei Ellenbogenextension gut zu erkennen.

\section{Frage 3}

Welcher dieser klinischen Tests wird nicht zur Diagnose der distalen Bizepssehnenruptur eingesetzt?
A Biceps-Crease-Test
B Supination-Pronation-Test
C Hook-Test
D Bizeps-Squeeze-Test
E Jerk-Test

\section{Frage 4}

Welche Aussage bezüglich Partialrupturen der distalen Bizepssehne ist richtig?

A Partialrupturen sind durch die spezifischen klinischen Tests gut diagnostizierbar.

B Bei der Inspektion fällt eine Retraktion des Muskels meist auf.

C Partialrupturen werden operativ therapiert, unabhängig vom Ausmaß der Ruptur.

D Ein alleiniges chirurgisches Débridement ist keine operative Option.

E Die Supinationsprüfung ist oft erst bei Ellenbogenflexion über $90^{\circ}$ schmerzhaft.

\section{Frage 5}

Wodurch ist die Bildgebung bei distaler Bizepssehnenruptur gekennzeichnet?

A Die ideale Position des Patientenarms zur Beurteilung des distalen Sehnenverlaufes im MRT ist die FABS-Position (Flexion, Abduktion und Supination).

B Die Ultraschalldiagnostik ist unmittelbar verfügbar, kostengünstig und untersucherunabhängig.

C Die Ultraschalldiagnostik kann das Insertionsgebiet am besten in voller Supination von posteroradial darstellen.

D Ein peritendinöses Ödem in der MRT-Bildgebung, bei sich ansonsten intakt darstellender Sehne, ist kein Indiz für eine Partialruptur.

E Eine konventionelle Röntgenaufnahme ist wenig hilfreich und sollte nicht durchgeführt werden.

- Weitere Fragen auf der folgenden Seite... 


\section{Punkte sammeln auf CME.thieme.de}

Fortsetzung $\ldots$

\section{Frage 6}

Eine der Aussagen bezüglich den Fixationstechniken bei der Refixation der distalen Bizepssehne ist falsch. Welche?

A Die höchste maximale Versagenslast in biomechanischen Studien hat der Endobutton.

B Radiusfrakturen im Bereich der Refixierungen sind eine seltene, jedoch mögliche Komplikation.

C Beim Anlegen eines Bohrkanals ist bei bikortikaler Bohrung eine Verletzung des N. interosseus posterior möglich.

D Die extraanatomische Fixationstechnik durch Annähen der distalen Bizepssehne an die Sehne des M. brachialis wird oft eingesetzt.

E Zur Refixierung der Sehne gibt es anatomische und extraanatomische Methoden.

\section{Frage 7}

Welche Aussage bezüglich der Operationstechniken zur Refixierung der distalen Bizepssehne ist falsch?

A Aufgrund von minimalinvasiven Methoden konnte das Risiko neurovaskulärer Verletzungen gesenkt werden.

B Es besteht kein Konsens hinsichtlich des postoperativen Managements.

C Bei der Single-Inzisions-Technik erfolgt der Zugang rein dorsal.

D In chronischen Fällen ist die Anatomie oft durch Narbengewebe deutlich verändert.

E In der Literatur ergeben sich keine relevanten Unterschiede beim Vergleich der Ergebnisse nach Single- vs. Double-Inzisions-Technik.

\section{Frage 8}

Wodurch ist die Versorgung chronischer distaler Bizepssehnenrupturen gekennzeichnet?

A Lediglich die Versorgung mittels Graft-Rekonstruktion steht zur Verfügung.

B Auch Refixationen in mehr als $60^{\circ}$ Flexion zeigen bezüglich Funktion und Bewegungsumfang sehr gute Ergebnisse, da ein deutliches Stretching des verkürzten Muskels möglich ist.

C Konservative und operative Ergebnisse sind bei der Behandlung von chronischen Rupturen vergleichbar.

D Die Versorgung mittels Sehnengraft-Interponat zeigt schlechte Ergebnisse.

E Ab 3 Monaten post Trauma spricht man von chronischen Rupturen.

\section{Frage 9}

Welche Aussage zum Erleiden einer distalen Trizepssehnenruptur ist richtig?

A Steroide senken das Risiko einer Ruptur.

B Gewisse endokrinologische systemische Erkrankungen können für eine Ruptur prädisponieren.

C Nicht körperlich tätige Frauen sind öfter betroffen als aktive Männer.

D Eine distale Trizepssehnenruptur kann in der Regel nicht klinisch festgestellt werden.

E Akute distale Trizepssehnenrupturen werden selten verpasst.

\section{Frage 10}

Eine der Aussagen zur Therapie der distalen Trizepssehnenruptur ist falsch. Welche?

A Die meisten distalen Trizepssehnenrupturen werden operativ behandelt.

B Bei der Refixation scheint ein Double-Row-Repair stabiler zu sein als ein Single-Row-Repair.

C Klinische Ergebnisse unterscheiden sich je nach Refixationstechnik.

D Die Hybridtechnik mittels transossärer Nähte und Anker ist der alleinigen transossären Naht in biomechanischen Studien nicht überlegen.

E Partialrupturen können unter konservativer Therapie auch bei Patienten mit hohem funktionellem Anspruch gute Ergebnisse zeigen. 